\title{
Dynamics of one- and two-dimensional fronts in a bistable equation with time-delayed global feedback: Propagation failure and control mechanisms
}

\author{
Yassine Boubendir* \\ Department of Mathematical Sciences, New Jersey Institute of Technology, University Heights, Newark, New Jersey 07102, USA \\ Vicenç Méndez \\ Department de Física Grup de Física Estadística, Universitat Autònoma de Barcelona, 08193 Bellaterra, Barcelona, Spain
}

Horacio G. Rotstein ${ }^{\dagger}$

Department of Mathematical Sciences, New Jersey Institute of Technology, Newark, New Jersey 07102, USA

(Received 16 May 2010; published 2 September 2010)

\begin{abstract}
We study the evolution of fronts in a bistable equation with time-delayed global feedback in the fast reaction and slow diffusion regime. This equation generalizes the Hodgkin-Grafstein and Allen-Cahn equations. We derive a nonlinear equation governing the motion of fronts, which includes a term with delay. In the onedimensional case this equation is linear. We study the motion of one- and two-dimensional fronts, finding a much richer dynamics than for the previously studied cases (without time-delayed global feedback). We explain the mechanism by which localized fronts created by inhibitory global coupling loose stability in a Hopf bifurcation as the delay time increases. We show that for certain delay times, the prevailing phase is different from that corresponding to the system in the absence of global coupling. Numerical simulations of the partial differential equation are in agreement with the analytical predictions.
\end{abstract}

DOI: 10.1103/PhysRevE.82.036601

PACS number(s): 05.45.Yv, 64.60.-i, 11.10.Lm

\section{INTRODUCTION}

The effects of global coupling (or feedback) and other types of nonlocal interactions on pattern formation have been studied in chemical [1-18], physical [19-25], and biological [26-31] spatially extended systems. Often, the resulting patterns are localized structures consisting of spatial domains, or clusters, of one type of dynamical behavior embedded in a background of another type separated by either static or moving boundaries. Typical examples are homogeneous clusters in different steady states, clusters oscillating out of phase, and clusters oscillating with different amplitudes. We refer the reader to the following reviews [32,33] on dissipative localized structures and solitons for further information. Localized structures with two clusters in different homogeneous steady states, or phases, arise in the bistable reactiondiffusion equation

$$
\epsilon \phi_{t}=\epsilon^{2} \Delta \phi+f(\phi)+\epsilon h
$$

defined in a domain $\Omega \in \mathbf{R}^{n}(n=1,2)$ with a global feedback term proportional to

$$
\langle\phi\rangle=\frac{1}{|\Omega|} \int_{\Omega} \phi(x, t) d x
$$

added to the right-hand side of Eq. (1) where $|\Omega|$ is the size of the domain [8] (see also [25]). In Eq. (1), $\phi$ is an order parameter, $\epsilon \ll 1$ (fast reaction and slow diffusion limit), and

\footnotetext{
*Also at Center for Applied Mathematics and Statistics (CAMS), NJIT.

${ }^{\dagger}$ Also at Center for Applied Mathematics and Statistics (CAMS), NJIT; horacio@njit.edu;
}

$f$ is a cubiclike function; i.e., a real odd function with a positive maximum, a negative minimum, and precisely three zeros located at $\phi_{-}, \phi_{0}$ and $\phi_{+}$respectively with $f^{\prime}\left(\phi_{ \pm}\right)<0$ and $f^{\prime}\left(\phi_{0}\right)>0$ [Fig. 1(a)]. We assume that $f$ is "minus" the derivative of a double-well potential having two equal minima at $\phi_{ \pm}$. For simplicity, and without loss of generality, here we consider $\phi_{ \pm}= \pm 1$ and $\phi_{0}=0$. The prototype example is $f(\phi)=\left(\phi-\bar{\phi}^{3}\right) / 2$. The constant $h$ specifies the difference between the potential local minima of the system; i.e., when $h \neq 0$ the double-well potential has two minima: one local and one global.

When $h=0$ (no potential difference between the two minima of the double well) Eq. (1) has two homogeneous stationary solutions: $\phi=\phi_{+}=+1$ or $\phi=\phi_{-}=-1$. We refer to them as the phases of the system. In a front solution for Eq. (1), the domain $\Omega$ is divided into two subdomains (clusters) $\Omega_{+}$and $\Omega_{-}$, where $\phi=\phi_{+}=+1$ and $\phi=\phi_{-}=-1$ respectively, separated by a very thin interface $\Gamma$ of width $\mathcal{O}(\epsilon)(\epsilon \ll 1)$ inside which $\phi$ varies rapidly between the values corresponding to the two phases $(\phi= \pm 1)[34]$ as we show schematically in Fig. 1(b) (left panel). The motion of the front represents a transition from one phase to the other. We use the convention adopted in Fig. 1: for $h>0$ the function $f(\phi)$ moves upward and the front moves to the left causing a phase transition from $\phi=\phi_{-}=-1$ to $\phi=\phi_{+}=+1 \quad\left(\phi_{-} \rightarrow \phi_{+}\right.$ phase transition). In a stationary, or localized front solution the two phases coexist. The evolution of fronts in spatially extended bistable systems has been extensively studied [35] (see also references therein). We review some points relevant to this paper at the end of this section.

The bistable equation has been used to describe phasetransition dynamics and the evolution of chemical concentrations $[35,36]$. In the former case, it is known as the AllenCahn equation [36]. The phases $\left(\phi_{ \pm}\right)$represent different 
(a)
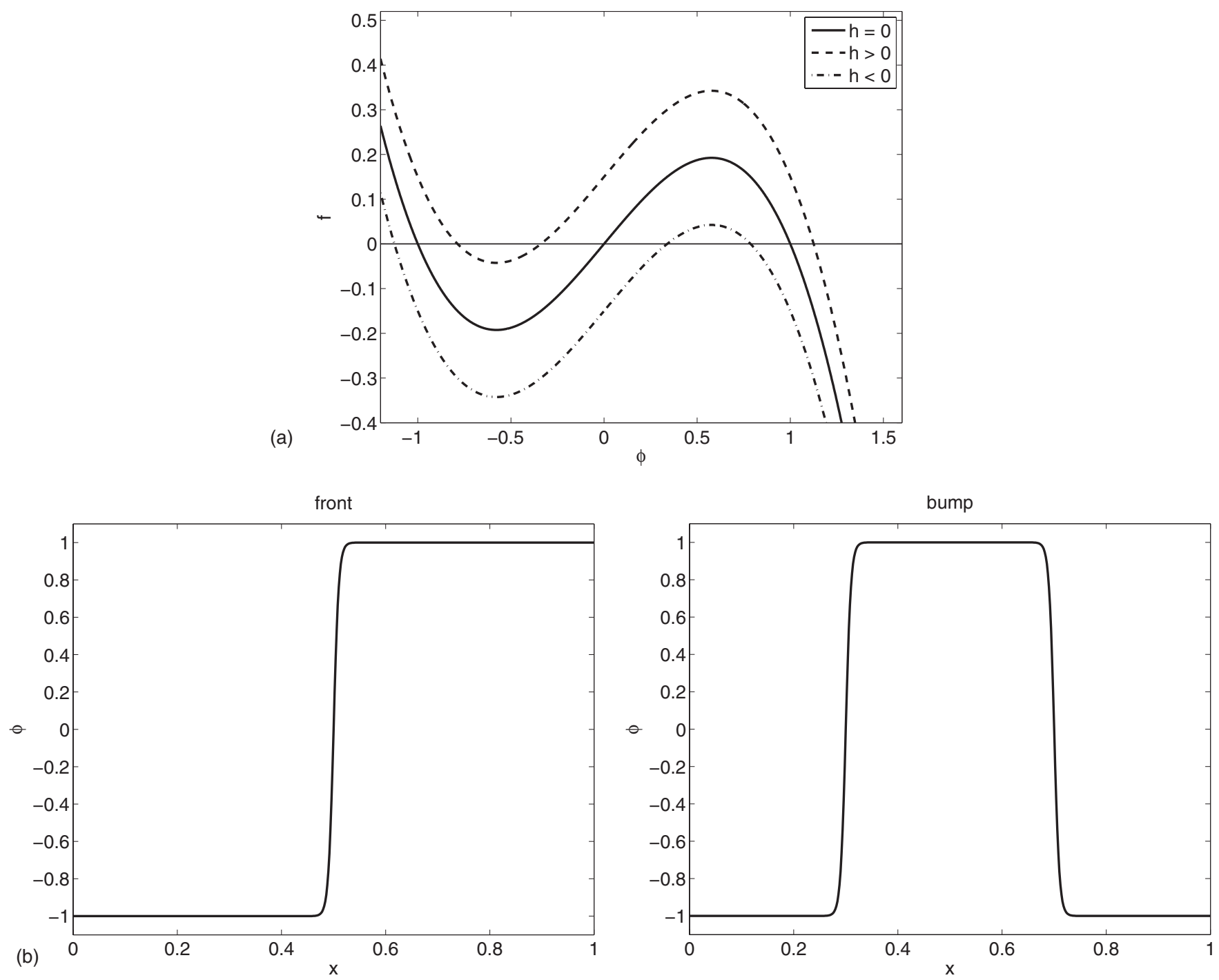

FIG. 1. (a) Schematic graph of a bistable function $f(\phi)+h$ for various values of $h$. The zeros of $f(\phi)+h\left(\phi_{-}, \phi_{0}\right.$ with $\phi_{+}$from left to right) are local extrema of the double-well potential function $F(\phi)-h \phi$ satisfying $f(\phi)=-F^{\prime}(\phi)$. The prototype example is $f(\phi)=\left(\phi-\phi^{3}\right) / 2$ with $F(\phi)=\phi^{4} / 8-\phi^{2} / 4$. The area above (below) the curve between $\phi_{0}$ and $\phi_{+}\left(\phi_{-}\right)$is proportional to the difference in heights between the maxima and the corresponding minimum of the double-well potential function. If the sign of the integral $I_{F}$ of the bistable function between $\phi_{-}$to $\phi_{+}$is positive (negative), then $\phi_{+}\left(\phi_{-}\right)$is the absolute minimum. The bistable function $f(\phi)$ is symmetric and has three zeros: $\phi_{-}=-1, \phi_{0}=0$, and $\phi_{+}=1$; i.e., $F(\phi)$ is also symmetric and has two local (and also absolute) minima: $\phi_{-}$and $\phi_{+}$. For $h>0, I_{F}>0$ and $\phi_{+}$ is the absolute minimum of the double-well potential $F(\phi)-h \phi$. For $h<0, I_{F}<0$ and $\phi_{-}$is the absolute minimum of the double-well potential $F(\phi)-h \phi$. (b) Schematic diagrams of a one-dimensional front (left) and bump (right).

chemical species or aggregation states of the system. In the context of brain dynamics the bistable equation is known as the Hodgkin-Grafstein model, and has been used as an early model to describe the evolution of extracellular potassium ions in the investigation of spreading depression in the brain [37] (see also [38]). The two phases correspond to a physiological and pathological states respectively [30,38].

The goal of this paper is to study the effects of delayedtime global feedback (or coupling) on the dynamics of fronts for the one- and two-dimensional bistable equation

$$
\epsilon^{2} \phi_{t}=\epsilon^{2} \Delta \phi+f(\phi)+\epsilon h+\epsilon \gamma\left\langle\phi_{\tau}\right\rangle,
$$

where $\left\langle\phi_{\tau}\right\rangle=\langle\phi\rangle(t-\tau)$ and where the constants $\gamma$ and $\tau$ are the global feedback parameter (intensity) and delay-time, re- spectively. We consider Neumann boundary conditions. All the variables and parameters considered here are assumed to be dimensionless. An appropriate non-dimensionalization can be performed to reduce dimensional cases to the one considered here. (See $[39,40]$ for a dimensional formulations of related problems.) We use formal asymptotic analysis, dynamical system tools and numerical simulations to investigate both the effects of the delay time $\tau$ on the dynamics of localized fronts generated as a result of instantaneous $(\tau=0)$ inhibitory global feedback $(\gamma<0)[8,40]$ and the role that delayed-time global feedback may play as a control mechanism for the selection of a resulting stationary homogenous phase. More specifically, in the absence of global inhibition $(\gamma=0)$ fronts evolve toward a homogeneous stationary solution with either $\phi=\phi_{+}$or $\phi=\phi_{-}$. We refer to the 
corresponding phase as the natural phase of the system. Stationary fronts are created for appropriate levels of instantaneous global inhibition $(\gamma<0$ and $\tau=0)$ but this alone is not enough to reverse the phase of the system; i.e., to create a homogeneous solution with a phase different from the natural one.

Time-delayed feedback, either local or global in space, arises in a variety of fields [41]. It has been used to control pattern forming systems [42-45], the emergence of traveling pulses in excitable media [30], chemical turbulence [46], rapid directional solidification [47], and chaos [48,49]. It has also been used to investigate networks of bistable oscillators [50,51], laser dynamics [52], and the spontaneous motion of cavity solitons [53]. We refer the reader to [41] for further applications. (See also [54]).

In bistable systems, time-delayed feedback, both local and global in space, arise in the context of brain dynamics $[30,38,55]$. Local time-delayed feedback has been investigated in the bistable equation [56], a bistable piecewise linear equation [57], and an extended version of the HodgkinGrafstein model of FitzHugh-Nagumo type. Time-delayed global feedback arises in the context of neural field dynamics [55] where the order parameter (neural field) captures the neural population activity. In Eq. (2.12) in [55] the timedelay parameter is space dependent and the reaction term is linear since this investigation focused on the stability of the rest state [58]. However, bistable functions have been used in spatially extended globally coupled models of neural dynamics where the neural activity is measured in terms of firing rate $[59,60]$. To our knowledge, the effects of delayed-time global feedback on the evolution of fronts in bistable systems have not been investigated before.

\section{A. Background}

One-dimensional fronts are also referred to as kinks. Standard singular perturbation arguments show that Eq. (3) with $\gamma=0$ (no global feedback) possesses a traveling kink moving with velocity proportional to $h[34,35]$. Hence, no localized solutions exist unless $h=0$ [61]. For the prototype bistable function $f(\phi)=\left(\phi-\phi^{3}\right) / 2$ and $h=0$ (no potential difference between phases) the localized kink is given by $\phi_{s}(x)= \pm \tanh \left[\left(x-x_{0}\right) /(2 \epsilon)\right]$, which is the solution of $\epsilon^{2} \phi_{x x}+f(\phi)=0$ satisfying $\phi_{s}\left(x_{0}\right)=0$. Note that the size of the interface decreases with $\epsilon$. If $h \neq 0$, then the kink propagates from the locally stable minimum to the globally stable minimum of the double-well potential [34].

In the two-dimensional (2D) case there exist front solutions, called quasi-2D kinks, which are locally kinks along normal directions to the interface $\Gamma$ [34]. For every time $t$ this interface can be approximated by the set of points $s(x, t)$ for which the order parameter $\phi$ vanishes. Note that $\Gamma(t ; \epsilon)=s(x, t)+\mathcal{O}(\epsilon)$ and $|\Omega|=\left|\Omega_{+}\right|+\left|\Omega_{-}\right|+\mathcal{O}(\epsilon)$ for appropriate size of $\left|\Omega_{+}\right|$.

The evolution of this interface characterizes the evolution of the corresponding front. These two terms will be used indistinctly in this paper. For $\gamma=h=0$ (no global feedback and no potential difference between phases), planar curved fronts move with normal velocity proportional to their cur- vature, according to the flow by mean curvature (FMC) equation $[36,62]$

$$
\frac{s_{t}}{\left(1+s_{x}^{2}\right)^{1 / 2}}=\frac{s_{x x}}{\left(1+s_{x}^{2}\right)^{3 / 2}}-h .
$$

In eq. (4), the left-hand represents the normal velocity and the first term in the right-hand represents the mean curvature. For $1 \mathrm{D}$ fronts, Eq. (4) reduces to $s_{t}=-h$ consistently with the results mentioned above. For a circular interface and $h=0$ (no potential difference between phases), the curvature is the reciprocal of the radius $R$, and Eq. (4) becomes $R_{t}=-R^{-1}(t)$, whose solution is given by $R(t)=\sqrt{R_{0}^{2}-2 t}$ for any initial condition $R_{0}$; i.e., circles shrink to a point at a critical finite time $t_{c}=R_{0}^{2} / 2$. For $h>0$ the value of $t_{c}$ decreases while for $h<0$ there exists a critical value $h_{c}$ such that circles either shrink to a point in finite time $\left(h>h_{c}\right)$ or grow unboundedly $\left(h<h_{c}\right)$; i.e., the potential difference between phases and the curvature effects compete, and the "winning" phase is determined by the initial radius. A qualitative similar behavior occurs for closed, convex interfaces; they remain convex and become asymptotically circular as they shrink to a point in finite time [63]. See also [64] for more general results.

Eq. (3) with instantaneous global inhibitory feedback $(\gamma<0$ and $\tau=0)$ possesses stationary fronts $[8,65]$. (See also [40] for the overdamped nonlinear wave equation.) The simplest argument for the 1D case is that the stationary kink solution $\phi_{s}(x)$ of $\epsilon^{2} \phi_{x x}+f(\phi)=0$ is also a solution of $\epsilon^{2} \phi_{x x}+f(\phi)+\epsilon(h+\gamma\langle\phi\rangle)$ provided $h+\gamma\langle\phi\rangle=0 \quad$ [65]. This heuristic argument remains true for the $2 \mathrm{D}$ case but it does not predicts the location of the interface.

\section{B. Overview}

In Sec. II, we use standard formal asymptotic techniques to derive an equation of motion that governs the evolution of fronts for Eq. (3). This equation generalizes the classical FMC Eq. (4) to include time-delayed global feedback effects. In Sec. III, we study the dynamics of one-dimensional fronts. We show how oscillatory fronts develop as delay time increases for $\gamma<0$ (inhibitory global feedback). They either stabilize into stationary fronts or, for large enough values of $\tau$, they grow unboundedly eventually reaching one of the boundaries of the domain. In Sec. IV, we study the dynamics of two-dimensional fronts with radial symmetry. For curved fronts, there is a competition between curvature and delayedtime global feedback effects. For the appropriate parameter values, the latter oppose shrinkage of curved fronts due to curvature effects thus creating either localized solutions or oscillatory fronts with growing amplitude which eventually cause phase reversal. The asymptotic techniques we use here have been applied before to investigate bistable systems in the absence of delayed-time global feedback. However, it is not clear a priori that the resulting equation of motion provides a good approximation to the front dynamics for Eq. (3) when delayed-time global feedback effects are included. In order to show that our results are not an artifact of these techniques we compare them with the results of numerical simulations using Eq. (3). We discuss the implications of our findings for pattern formation in Sec. V. 


\section{DERIVATION OF THE EQUATION OF FRONT MOTION}

Here we follow other authors [8,34,66,67] and use formal asymptotic analysis to derive an equation that describes the evolution of a fully developed front. Although some of the arguments are standard for systems with no delayed-time global feedback we include them here for completeness. We assume that for small $\epsilon \geq 0$ and all $t \in[0, T]$, the domain $\Omega$ can be divided into two open regions $\Omega_{+}(t ; \epsilon)$ and $\Omega_{-}(t, \epsilon)$ by a curve $\Gamma(t ; \epsilon)$, which does not intersect $\partial \Omega$. This interface, defined by

$$
\Gamma(t ; \epsilon):=\{x \in \Omega: \phi(x, t ; \epsilon)=0\},
$$

is assumed to be smooth, which implies that its curvature and its velocity are bounded independently of $\epsilon$. We also assume that there exists a solution $\phi(x, t ; \epsilon)$ of Eq. (3), defined for small $\epsilon$, for all $x \in \Omega$ and for all $t \in[0, T]$ with an internal layer. As $\epsilon \rightarrow 0$ this solution is assumed to vary continuously through the interface, taking the value 1 when $x \in \Omega_{+}(t ; \epsilon)$, -1 when $x \in \Omega_{-}(t, \epsilon)$, and varying rapidly but smoothly through the interface. By carrying out a singular perturbation analysis for $\epsilon \ll 1$, we obtain the law of motion of the interface, treating it as a moving internal layer of width $\mathcal{O}(\epsilon)$. We focus on the dynamics of the fully developed layer, and not on the process by which it was generated.

The asymptotic expansion for $\phi$ away from a small, $\mathcal{O}(\epsilon)$, neighborhood of the interface is assumed to have the form $\phi \sim \phi^{0}+\epsilon \phi^{1}+\mathcal{O}\left(\epsilon^{2}\right)$ as $\epsilon \rightarrow 0$. Substituting into Eq. (3) and equating coefficients of the corresponding powers of $\epsilon$ we obtain the following $\mathcal{O}(1)$ and $\mathcal{O}(\epsilon)$ problems for the outer solution: $\quad f\left(\phi^{0}\right)=0$ and $f^{\prime}\left(\phi^{0}\right) \phi^{1}+h+\gamma\left\langle\phi_{\tau}^{0}\right\rangle=0$. The corresponding solutions are $\phi^{0}= \pm 1 \quad\left(x \in \Omega_{ \pm}\right)$and $\phi^{1}=-\left[h+\gamma\left|\Omega_{+}\right|(t-\tau)-\gamma\left|\Omega_{-}\right|(t-\tau)\right] / f^{\prime}\left(\phi^{0}\right), \quad$ respectively. Away from the interface the solution is given by the phases $\left(\phi_{0}= \pm 1\right)$ corrected by an $\mathcal{O}(\epsilon)$ term.

We now look at the inner solution in the interface of width $\mathcal{O}(\epsilon)$. In Cartesian coordinates this interface is represented by $y=s(x, t, \epsilon)$. We assume that the curvature of the front is small compared to its width and define a new variable $z:=\frac{y-s(x, t, \epsilon)}{\epsilon}=\mathcal{O}(1)$ as $\epsilon \rightarrow 0$ in a neighborhood of the interface. (In the one-dimensional case $s=s(t ; \epsilon)$ and $y:=\frac{x-s(t, \epsilon)}{\epsilon}$.)

We call $\Phi(z, x, t, \epsilon)$ the asymptotic form of $\phi$ as $\epsilon \rightarrow 0$ with $z$ fixed. Equation (3) in $(z, x, t)$ coordinates becomes

$$
\begin{aligned}
\epsilon^{2} \Phi_{t}-\epsilon s_{t} \Phi_{z}= & \epsilon^{2} \Phi_{x x}-2 \epsilon s_{x} \Phi_{z x}+\left(1+s_{x}^{2}\right) \Phi_{z z}-\epsilon s_{x x} \Phi_{z}+f(\Phi) \\
& +\epsilon h+\epsilon \gamma\left\langle\phi_{\tau}\right\rangle .
\end{aligned}
$$

The asymptotic expansion of $\Phi$ is assumed to have the form $\Phi \sim \Phi^{0}+\epsilon \Phi^{1}+\mathcal{O}\left(\epsilon^{2}\right)$ as $\epsilon \rightarrow 0$. Substituting into Eq. (6) and equating coefficients of the corresponding powers of $\epsilon$ we obtain the following problems for $\mathcal{O}(1)$ and $\mathcal{O}(\epsilon)$ respectively,

$$
\begin{gathered}
\left(1+s_{x}^{2}\right) \Phi_{z z}^{0}+f\left(\Phi^{0}\right)=0 \\
\left(1+s_{x}^{2}\right) \Phi_{z z}^{1}+f^{\prime}\left(\Phi^{0}\right) \Phi^{1}=\left(s_{x x}-s_{t}\right) \Phi_{z}^{0}+2 s_{x} \Phi_{z x}^{0}-h-\gamma\left\langle\phi_{\tau}^{0}\right\rangle .
\end{gathered}
$$

In order to solve Eq. (7) we define a new variable

$$
\xi:=\frac{z}{\left(1+s_{x}^{2}\right)^{1 / 2}} .
$$

In terms of $\xi$, Eq. (7) reads

$$
\Phi_{\xi \xi}^{0}+f\left(\Phi^{0}\right)=0
$$

whose solution is $\Phi^{0}=\Psi(\xi)$, the unique solution of $\Psi^{\prime \prime}+f(\Psi)=0, \quad \Psi( \pm \infty)= \pm 1, \quad \Psi(0)=0$. Thus

$$
\Phi^{0}=\Phi^{0}\left(\frac{z}{\left(1+s_{x}^{2}\right)^{1 / 2}}\right) .
$$

Note that as $z$ moves away from zero $\Phi^{0}$ approaches $\phi^{0}= \pm 1$.

In terms of $\xi, x$ and $t$, Eq. (8) reads

$$
\begin{gathered}
\Phi_{\xi \xi}^{1}+f^{\prime}\left(\Phi^{0}\right) \Phi^{1}=\frac{s_{x x}-s_{t}}{\left(1+s_{x}^{2}\right)^{1 / 2}} \Psi^{\prime}- \\
2 \frac{s_{x}^{2} s_{x x}}{\left(1+s_{x}^{2}\right)^{3 / 2}}\left(\xi \Psi^{\prime \prime}+\Psi^{\prime}\right)-h-\gamma\left\langle\phi_{\tau}^{0}\right\rangle .
\end{gathered}
$$

It is straightforward to check that $\Psi^{\prime}(\xi)$ satisfies the homogeneous equation

$$
\Phi_{\xi \xi}^{1}+f^{\prime}\left(\Phi^{0}\right) \Phi^{1}=0 .
$$

Thus the operator $\Lambda:=\frac{\partial^{2}}{\partial \xi^{2}}+f^{\prime}\left(\Phi^{0}\right)$ has a simple eigenvalue at the origin with $\Psi^{\prime}$ as the corresponding eigenfunction. Then the solvability condition for the Eq. (12) gives

$$
\begin{aligned}
& \frac{s_{x x}-s_{t}}{\left(1+s_{x}^{2}\right)^{1 / 2}} \int_{-\infty}^{\infty}\left(\Psi^{\prime}\right)^{2} d \xi-2 \frac{s_{x}^{2} s_{x x}}{\left(1+s_{x}^{2}\right)^{3 / 2}} \\
& \quad \times \int_{-\infty}^{\infty}\left(\xi \Psi^{\prime \prime}+\Psi^{\prime}\right) \Psi^{\prime} d \xi-\left[h+\gamma\left\langle\phi_{\tau}^{0}\right\rangle\right] \int_{-\infty}^{\infty} \Psi^{\prime} d \xi=0 .
\end{aligned}
$$

A simple calculation shows that $\int_{-\infty}^{\infty} \xi \Psi^{\prime} \Psi^{\prime \prime} d \xi$ $=-\frac{1}{2} \int_{-\infty}^{\infty}\left(\Psi^{\prime}\right)^{2} d \xi$. Calling

$$
\alpha=\frac{\Psi(+\infty)-\Psi(-\infty)}{\int_{-\infty}^{\infty}\left(\Psi^{\prime}\right)^{2} d \xi}
$$

and rearranging terms we get

$$
\frac{s_{t}}{\left(1+s_{x}^{2}\right)^{1 / 2}}-\frac{s_{x x}}{\left(1+s_{x}^{2}\right)^{3 / 2}}+\alpha h+\alpha \gamma\left\langle\phi_{\tau}^{0}\right\rangle=0 .
$$

We know take care of the last term in Eq. (16). 


$$
\begin{aligned}
\int_{\Omega} \phi^{0}(x, t) d x= & \int_{\Omega^{+}(t)} \phi^{0}(x, t) d x+\int_{\Omega^{-}(t)} \phi^{0}(x, t) d x \\
& +\int_{\Gamma} \phi^{0}(x, t) d x \\
& =\int_{\Omega^{+}(t)} \phi^{0}(x, t) d x+\int_{\Omega^{-}(t)} \phi^{0}(x, t) d x+\mathcal{O}(\epsilon) \\
& =\Omega^{+}(t)-\left|\Omega^{-}(t)\right|+\mathcal{O}(\epsilon) \\
& =\left(|\Omega|-2\left|\Omega_{-}(t)\right|\right)+\mathcal{O}(\epsilon) .
\end{aligned}
$$

Substituting Eq. (17) into Eq. (16) and rearranging terms we get

$$
\frac{s_{t}}{\left(1+s_{x}^{2}\right)^{1 / 2}}=\frac{s_{x x}}{\left(1+s_{x}^{2}\right)^{3 / 2}}-\alpha h-\frac{\alpha \gamma}{|\Omega|}\left(|\Omega|-2\left|\Omega^{-}\right|(t-\tau)\right)=0 .
$$

Eq. (18) governs the evolution of fronts for Eq. (3) and generalizes the classical FMC Eq. (4) to include delayed global feedback effects. Note that the constant $\alpha$ given by Eq. (15) depends on the function $f(\phi)$ with $\Psi(\xi)$ the unique solution of $\Psi^{\prime \prime}+f(\Psi)=0, \Psi( \pm \infty)= \pm 1, \Psi(0)=0 . \quad$ For the prototype case $f(\phi)=\left(\phi-\phi^{3}\right) / 2$ (Ginzburg-Landau theory), $\Psi(\xi)=\tanh \frac{\xi}{2}$ and $\alpha=3$. Note that for $f(\phi)=\sin \phi$ (overdamped sine-Gordon equation), $\Psi(\xi)=4 \tan ^{-1} e^{\xi}-\pi$ and $\alpha=\frac{\pi}{4}$. For simplicity and without loss of generality, throughout this paper we will consider $|\Omega|=1$ and $\alpha=1$; i.e., the values of $|\Omega|$ and $\alpha$ are absorbed by the parameters $h$ and $\gamma$.

In polar coordinates Eq. (18) reads

$$
\begin{aligned}
& \rho \rho_{t}\left(\rho^{2}+\rho_{\theta}^{2}\right)+\rho^{2}-\rho \rho_{\theta \theta}+2 \rho_{\theta}^{2} \\
& \quad+\left[h+\gamma\left(1-2\left|\Omega^{-}\right|(t-\tau)\right]\left(\rho^{2}+\rho_{\theta}^{2}\right)^{3 / 2} .\right.
\end{aligned}
$$

Eq. (19) can be obtained from its corresponding Cartesian version [Eq. (18)] by changing variables or it can be derived directly from Eq. (3) in a similar fashion as for the Cartesian version presented in Sec. II. (See [66] for technical details).

\section{DYNAMICS OF ONE-DIMENSIONAL FRONTLIKE STRUCTURES}

The evolution of a one-dimensional front in a domain $\Omega=[0,1]$ is given by following linear delay-differential equation

$$
s_{t}=-h-\gamma[1-2 s(t-\tau)] .
$$

Equation (20) is obtained from Eq. (18) by disregarding spatial variations $\left(s_{x}=s_{x x}=0\right)$. A schematic diagram of a front is given in Fig. 1(b) (left).

As mentioned in Sec. I (Background), for $\gamma=0$ (no global feedback) the sign of $h$ governs the dynamics of the front and determines the homogeneous steady phase. For $h \neq 0$, no localized solutions are possible. If $h>0$, the zero of the bistable function $f(\phi)+\epsilon h$ is moved to the right [see Fig. 1(a)] and, to first order approximation, the phase $\phi_{+}=+1$ becomes an absolute minimum of the corresponding double- well potential; i.e., a front as the one shown in Fig. 1(b) (left panel) moves to the left, in a direction that favors the $\phi_{-} \rightarrow \phi_{+}$phase transition, eventually generating a homogeneous steady solution. Analogous results hold for the case $h<0$. In the context of neural dynamics fronts moving to the left $\left(\phi_{-} \rightarrow \phi_{+}\right)$are referred to as depolarizing and fronts moving in the opposite direction are referred to as nondepolarizing (or hyperpolarizing). In the context of solid-liquid phasetransition dynamics, the former are referred to as solidification fronts.

Localized solutions for Eq. (20) exist for parameter values such that the fixed point $\bar{s}=1 / 2+h /(2 \gamma)$ is stable and satisfies $0<\bar{s}<1$. The latter constraint, imposed by the endpoints of the domain $\Omega$, yields $-1<h / \gamma<1(|\gamma|>|h|)$. When there is no delay $(\tau=0), \bar{s}$ is stable for $\gamma<0$ (global inhibition) and unstable for $\gamma>0$. Hence, localized solutions are created as the result of global inhibition (and not global excitation). Fronts initially away from $\bar{s}$ converge to $\bar{s}$ and cease to move. As global inhibition increases ( $\gamma$ increases in absolute value), $\bar{s} \rightarrow 1 / 2$; i.e., localized solutions persist for all negative values of $\gamma$. Therefore, given $h$, instantaneous global inhibition is not enough to reverse the phase of the system. Below we show that phase reversal can be achieved as the delay time increases above a critical value through a Hopf-bifurcation mechanism.

For $\tau>0$ stability of $\bar{s}$ can be investigated following standard procedures [41]. We sketch the analysis in Appendix A for completeness. We show that stable localized solutions exist for values of $0<\tau<\tau_{H}=-\pi /(4 \gamma)$ and $\gamma<0$ (global inhibition), and they loose stability at the Hopf bifurcation point $\tau_{H}$. In addition, there is a critical value $\tau_{c}=\tau \leq(2 \gamma e)^{-1}, \tau_{c}<\tau_{H}$, such that upon perturbations to $\bar{s}$ front oscillations will be observed for $\tau_{c}<\tau<\tau_{H}$ and no oscillations will be observed for $\tau<\tau_{c}$.

In Fig. 2 we illustrate the evolution of one-dimensional fronts for $h=0, \gamma=-1$ and various representative values of $\tau$. For these values our asymptotic approximation predicts $\tau_{c} \sim 0.184$ and $\tau_{H} \sim 0.785$. The solid lines correspond to the solutions to the asymptotic Eq. (20). The superimposed circles correspond to the solution of the one-dimensional PDE [Eq. (3)] with $\epsilon=0.01$ (see below). Figure 2(a) shows the localized front corresponding to $\tau=0$ (no delay). For small values of $\tau<\tau_{c}$ the localized solutions persist [Fig. 2(b)]. For larger values of $\tau$ (but still $\tau<\tau_{H}$ ) damped oscillations develop which eventually converge to the localized solution $\bar{s}$ [Figs. 2(c)-2(e)]. For higher values of $\tau>\tau_{H}$, oscillations grow unboundedly [Fig. 2(f)] and eventually the front will reach one of the boundaries (either $s=0$ or $s=1$ ) leaving the domain in a homogeneous phase with either $\phi$ $=+1$ or $\phi=-1$, respectively. Appropriate values of $\gamma$ and $\tau$ can be used so that either a localized solution or a stationary solution with a desired phase is selected.

In Fig. 3(a), we present graphs of the frequency of the oscillatory fronts as a function of the delay-time $\tau$ for $h=0$ and three representative values of $\gamma$. The "Red curve" $(\gamma=-1)$ corresponds to Fig. 2. The arrows pointing downward above each curve indicate the Hopf-bifurcation delaytime $\tau_{H}$. For each value of $\gamma$, points left to the arrows correspond to stable oscillations while points right to the arrows correspond to unstable oscillations. The front's oscillatory 

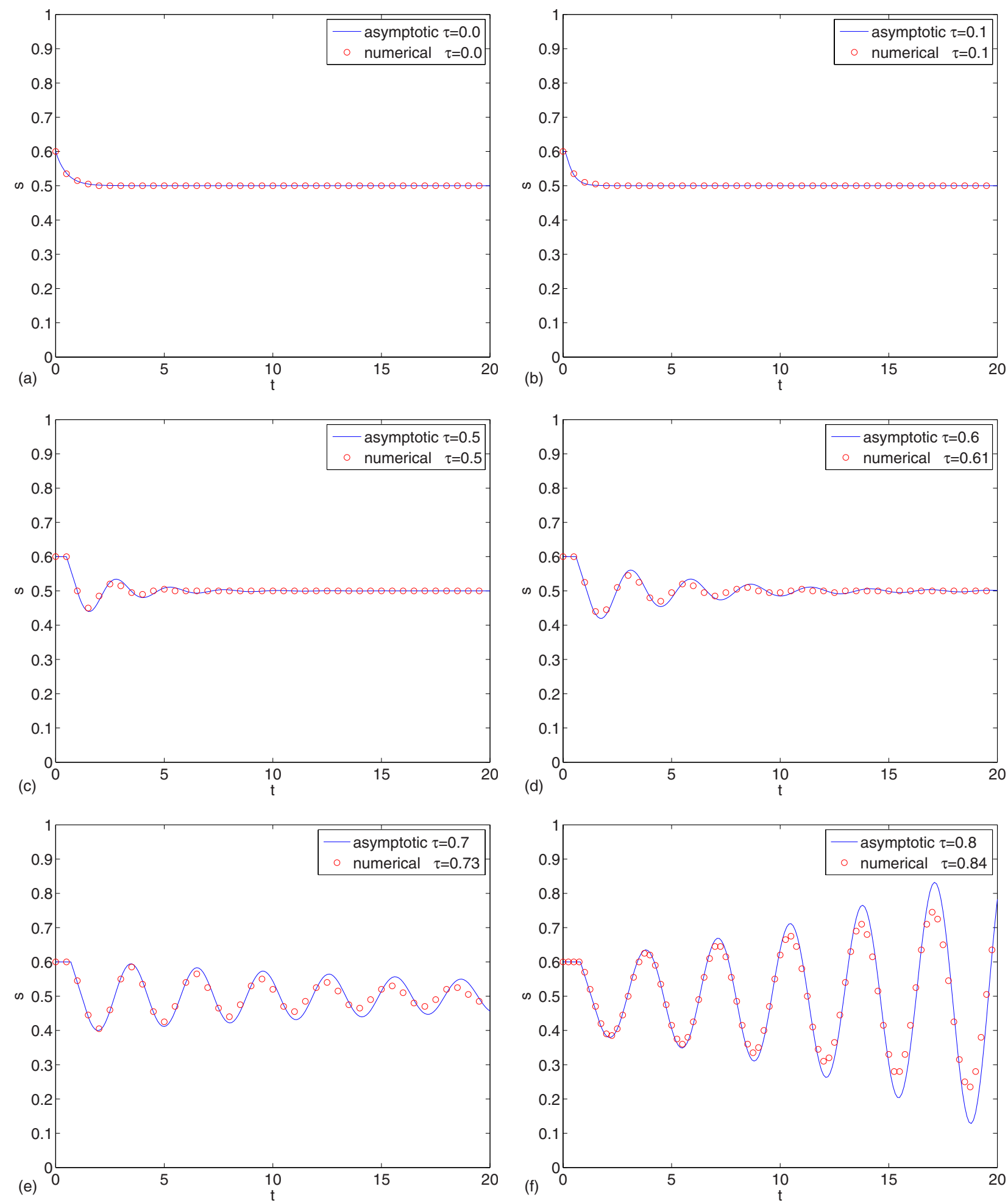

FIG. 2. (Color online) Evolution of one-dimensional fronts for $h=0, \gamma=-1$ and various values of $\tau$. For these values of $h$ and $\gamma, \tau_{H} \sim 0.785$ and $\tau_{c} \sim 0.184$. Solid lines correspond to the solution to the asymptotic Eq. (20). Superimposed circles correspond to the solution of the one-dimensional PDE [Eq. (3)] with $\epsilon=0.01$.

frequency is a decreasing function of $\tau$, for fixed-values of $\gamma$, and an increasing function of $\gamma$ for fixed values of $\tau$. The parameters $\gamma$ and $\tau$ can be finely tuned for the front to be on either side of the arrows. The "similarity" among the three curves in Fig. 3(a) is not surprising since eq. (20) can be rescaled to the following $\gamma$-independent equation

$$
\frac{d \hat{s}}{d \hat{t}}=-\hat{h}-1+2 \hat{s}(\hat{t}-\hat{\tau})
$$

by defining 

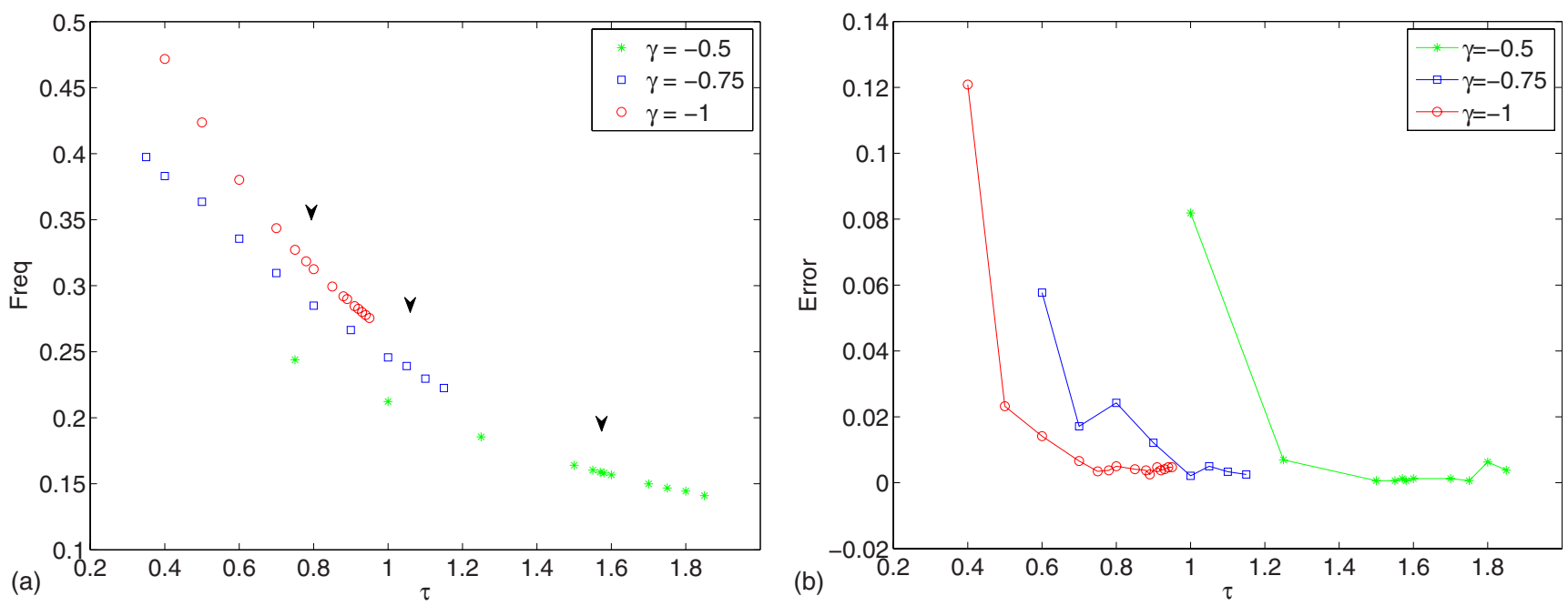

FIG. 3. (Color online) Frequency of the oscillatory one-dimensional fronts as a function of the delay-time $\tau$ for $h=0$ and various values of $\gamma$. The circles (light gray) graphs correspond to the solutions presented in Fig. 2. (a) Frequency of the oscillatory fronts for the asymptotic Eq. (20). The arrows indicate the values of $\tau_{H}$ corresponding to a Hopf-bifurcation. (b) Frequency error between the solutions to the asymptotic Eq. (20) and the PDE [Eq. (3)] as a function of $\tau$. For each value of $\tau$, the error was calculated as the absolute value of the difference between the two frequencies divided by the frequency corresponding to $\tau_{H}$.

$$
\hat{t}=\gamma t, \quad \hat{\tau}=\gamma \tau, \quad \hat{h}=\frac{h}{\gamma}, \quad \hat{s}(\hat{t})=s(\hat{t} / \gamma) .
$$

For Eq. (21), $\tau_{H}=\pi / 4$ (see Appendix A). The curves in Fig. 3(a) are representative for values of $h \neq 0$. Changes in the value of $h$ are reflected in the location of the equilibrium front $\bar{s}$ but not in the stability properties of the front solution $s(t)$ (see Appendix A).

In order to show that our findings are not an artifact of the asymptotic approximation, we performed direct simulations of Eq. (3) (one-dimensional case) for $\gamma=-1, h=0, \epsilon=0.01$ and various values of $\tau$ and compare with our asymptotic predictions. Our results are presented in Figs. 2-4. Numerical simulations of Eq. (3) were performed for the prototypical bistable function $f(\phi)=\left(\phi-\phi^{3}\right) / 2$ using a CrankNicholson finite difference scheme together with the Newton method for the nonlinear term [68]. We have considered a computational domain defined by $\Omega:=[0,1]$, a spatial discretization of 200 points, Neuman boundary conditions at $x=0$ and $x=1$, a time discretization $\Delta t=0.01$ (or sometimes $\Delta t=0.001)$, and the following initial condition $\phi(x)=\tanh \left(\frac{x-0.6}{\epsilon}\right)$. The results for representative values of $\tau$ and $\gamma=-1$ are presented in Fig. 4. The thin interfaces can be observed in between the "red" and "blue" regions corresponding to the two phases, $\phi=+$ and $\phi=-1$, respectively. These graphs illustrate the fact that the front structure of the solution is preserved for values of $\gamma<0$ and $\tau>0$ even when the front looses stability. In Fig. 2 we superimposed (circles) the values of $x$ approximating the interface $[\phi(x)=0]$ corresponding to the PDE Eq. (3) to the asymptotic front solution $s(t)$. The asymptotic prediction and the numerical solutions are in good agreement although the values of $\tau$ that give the best approximation between the asymptotic and numerical solutions slightly differ as $\tau$ increases.

Further comparisons between the asymptotic and numerical results are presented in Fig. 3(b). There we plotted the relative frequency error between the solutions to the asymptotic Eq. (20) and the numerical solutions to the PDE [Eq. (3)] as a function of $\tau$. For each value of $\gamma$ we calculated the relative frequency error as the absolute value of the difference between the two frequencies divided by the frequency corresponding to the Hopf-bifurcation delay-time $\tau_{H}$. Overall, there is good agreement between the asymptotic and numerical predictions; the relative error is below 0.02 . The error is larger for small values of $\tau$, slightly above the critical delay-time $\tau_{c}$ for the emergence of oscillatory solutions. These may not necessarily indicate a lack of accuracy in the asymptotic approximation but rather in the calculation of the frequency since for these values of $\tau$ the amplitude of the damped oscillations decreases very rapidly. This is further supported by the fact that the asymptotic prediction is very accurate for smaller values of $\tau$ as shown in Figs. 2(a)-2(d).

Deporalization spreading in two directions gives rise to bumps [see Fig. 1(b) (right)] which are characteristic in the dynamics of neural fields [69]. The evolution of bumps for Eq. (3) can be investigated by looking at the motion of two separate fronts $s_{1}$ and $s_{2}$ moving in opposite directions for the same value of $h$. The corresponding equations are

$$
\begin{gathered}
s_{1, t}=-h-\gamma\left[1-2\left|\Omega^{-}\right|(t-\tau) \mid\right], \\
s_{2, t}=h+\gamma\left[1-2\left|\Omega^{-}\right|(t-\tau) \mid\right],
\end{gathered}
$$

with $s_{1}(0)=s_{1,0}$ and $s_{2}(0)=s_{2,0}$, and where we assume without loss of generality that $s_{2,0}-s_{1,0}>0$. Since

$$
\left|\Omega^{-}\right|(t)=1+s_{1}(t)-s_{2}(t),
$$

eqs. (23) become

$$
\begin{gathered}
s_{1, t}=-h-\gamma\left[-1+2\left(s_{2}-s_{1}\right)(t-\tau) \mid\right], \\
s_{2, t}=h+\gamma\left[-1+2\left(s_{2}-s_{1}\right)(t-\tau) \mid\right] .
\end{gathered}
$$



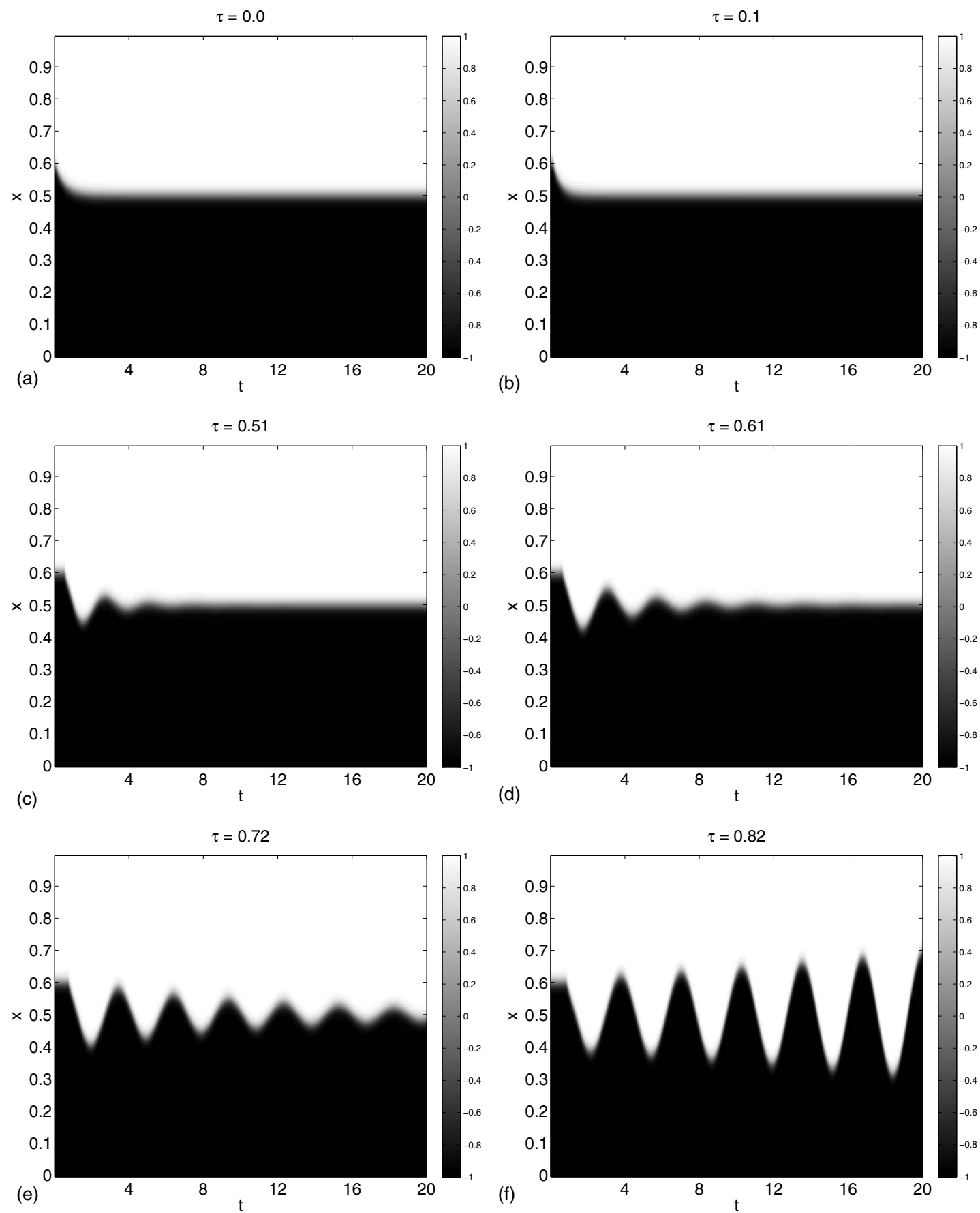

FIG. 4. Evolution of one-dimensional fronts for Eq. (3) for $h=0, \gamma=-1, \epsilon=0.01$ and various values of $\tau$. The gray level codes for values of $\phi$. The interface (or front) corresponds to the small region of fast transition between $\phi=+1$ (white) and $\phi=-1$ (black). The values of $\tau$ appearing at the top of each figure are approximation of the values used in our simulations: (a) $\tau=0.0$, (b) $\tau=0.1$, (c) $\tau=0.51$, (d) $\tau=0.61$, (e) $\tau=0.72$ and (f) $\tau=0.82$.

For $\gamma=0$ (no global feedback), the two fronts move in opposite directions. Following our previous discussion, if $h>0, s_{1}$ decreases and $s_{2}$ increases; i.e., the fronts move in a direction that favors the transition from $\phi=-1$ to $\phi=+1$, eventually generating a homogeneous solution. If $h<0$, the bump collapses (when the two fronts meet) and a homogeneous solution in the opposite phase is created.

For $\gamma \neq 0$ and $\tau=0$ (no delay), the solution to system [Eq. (24)] is given by

$$
\begin{aligned}
& s_{1}(t)=\frac{\gamma-h-2 \gamma\left(s_{2,0}-s_{1,0}\right)}{4 \gamma}\left(e^{4 \gamma t}-1\right)+s_{1,0}, \\
& s_{2}(t)=\frac{\gamma-h-2 \gamma\left(s_{2,0}-s_{1,0}\right)}{4 \gamma}\left(1-e^{4 \gamma t}\right)+s_{2,0} .
\end{aligned}
$$

A stable bump exists provided $\gamma<0$ (inhibitory global feedback). In this case 

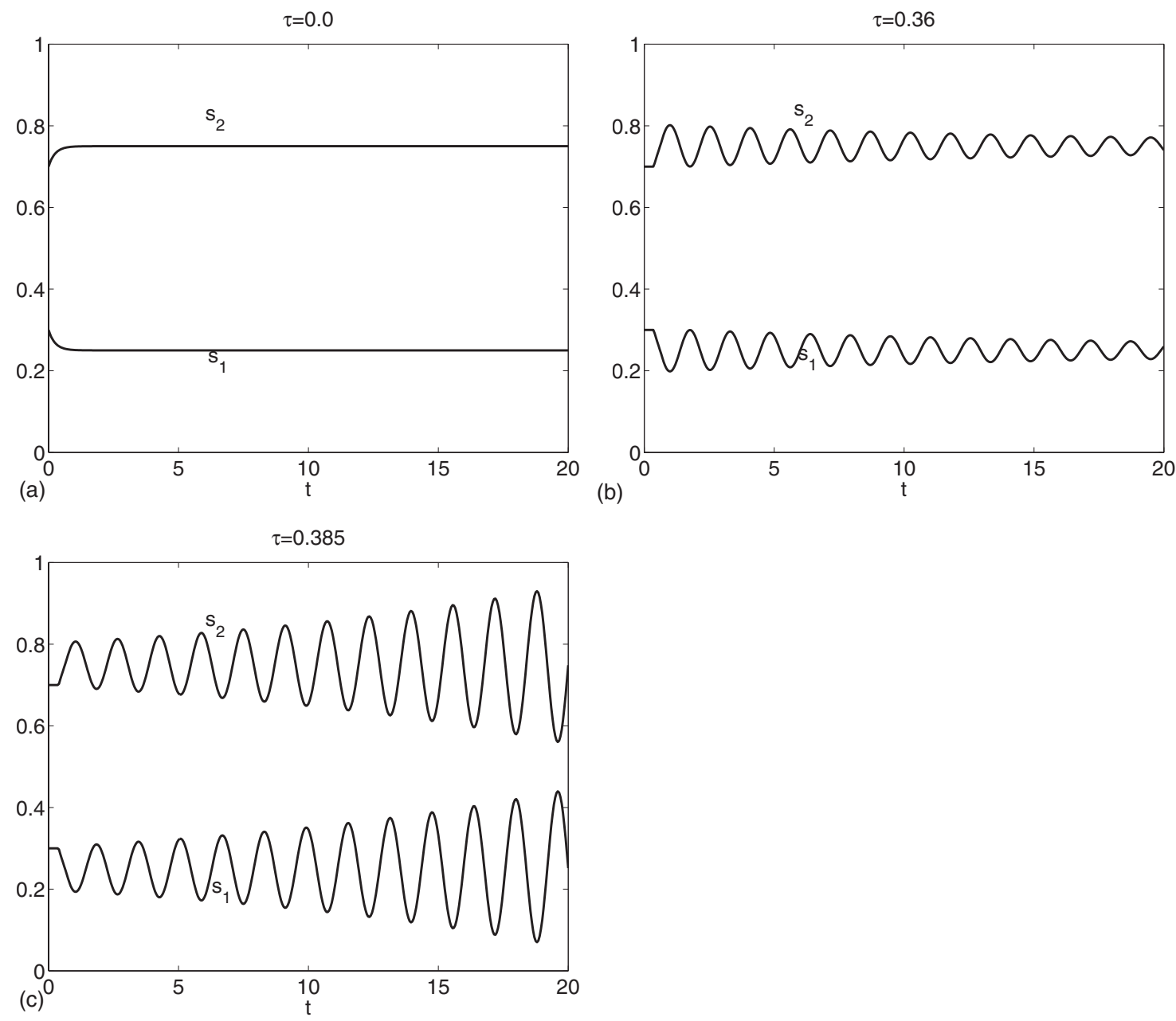

FIG. 5. Evolution of one-dimensional bumps according to Eq. (23) for $h=0, \gamma=-1$ and various values of $\tau$ : (a) 0.0 , (b) 0.36 , and (c) 0.385 .

$$
\lim _{t \rightarrow \infty} s_{1}(t)=-\frac{\gamma-h-2 \gamma\left(s_{1,0}+s_{2,0}\right)}{4 \gamma}
$$

similar to these in Fig. 5. We used the following initial conditions: $\phi(x)=\tanh \left(\frac{x-0.3}{\epsilon}\right) \tanh \left(\frac{0.7-x}{\epsilon}\right)$. As expected, the dynamics of bumps are qualitatively similar to the dynamics of fronts. Figure 5(a) shows a localized bump for $\tau=0$. For values of $\tau<\tau_{H}$, the localized bump persists. If front oscillations develop they decay in amplitude and the "oscillatory bump" eventually stabilizes [Fig. 5(b)]. For values of $\tau>\tau_{H}$ [Fig. 5(c)], oscillations grow in amplitude. Depending on the values of $\gamma$ and $\tau$, the bump may collapse or reach the boundaries of the domain thus creating homogeneous solutions. The prevailing phases are $\phi=-1$ and $\phi=1$ in the former and latter cases respectively as we show in Figs. 6(d) and 6(e). These figures also show that the bump structure of the solutions is preserved in the PDE even for values of $\tau$ for which these bumps loose stability.

Comparison between Figs. 5 and 6 show a good agreement between the analytical and numerical predictions. As for fronts, in Fig. 6 we have chosen values of $\tau$ that produce graphs qualitatively similar to the ones in Fig. 5 (i.e., the best approximation). Then we compared the values of $\tau$ which coincide ( $\tau=0$ and differ by 0.02 and 0.035 in the other two graphs). As it occurs for fronts, these results are representative for other values $h$ and $\gamma$. 


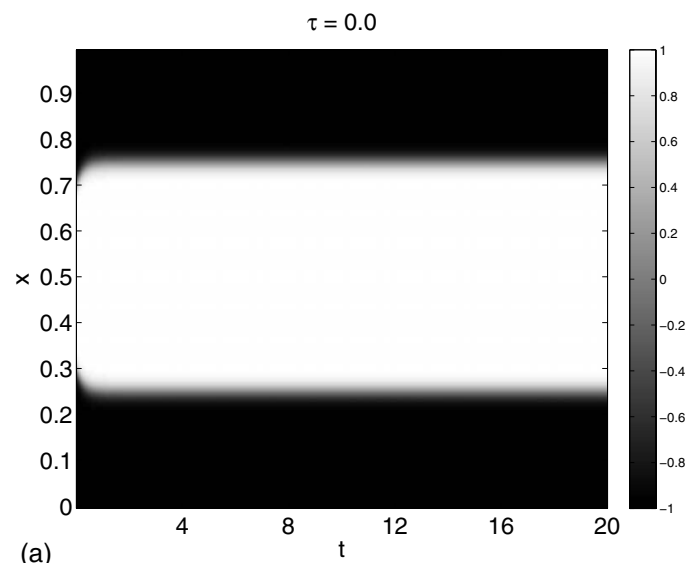

(a)

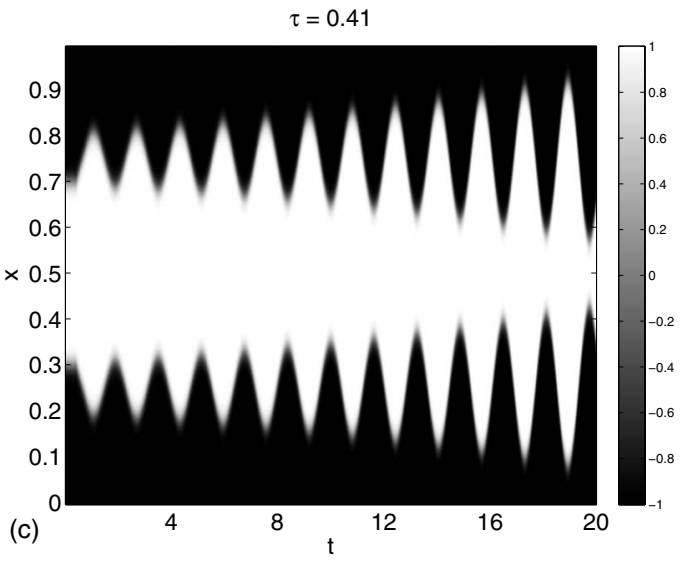

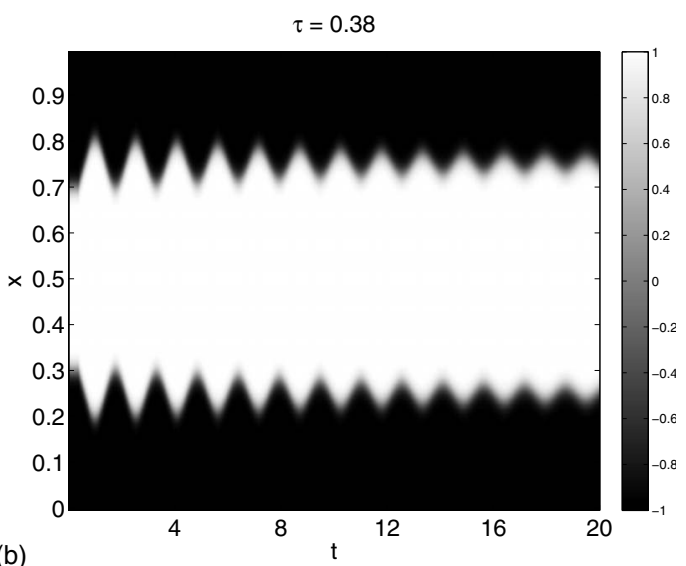

(b)

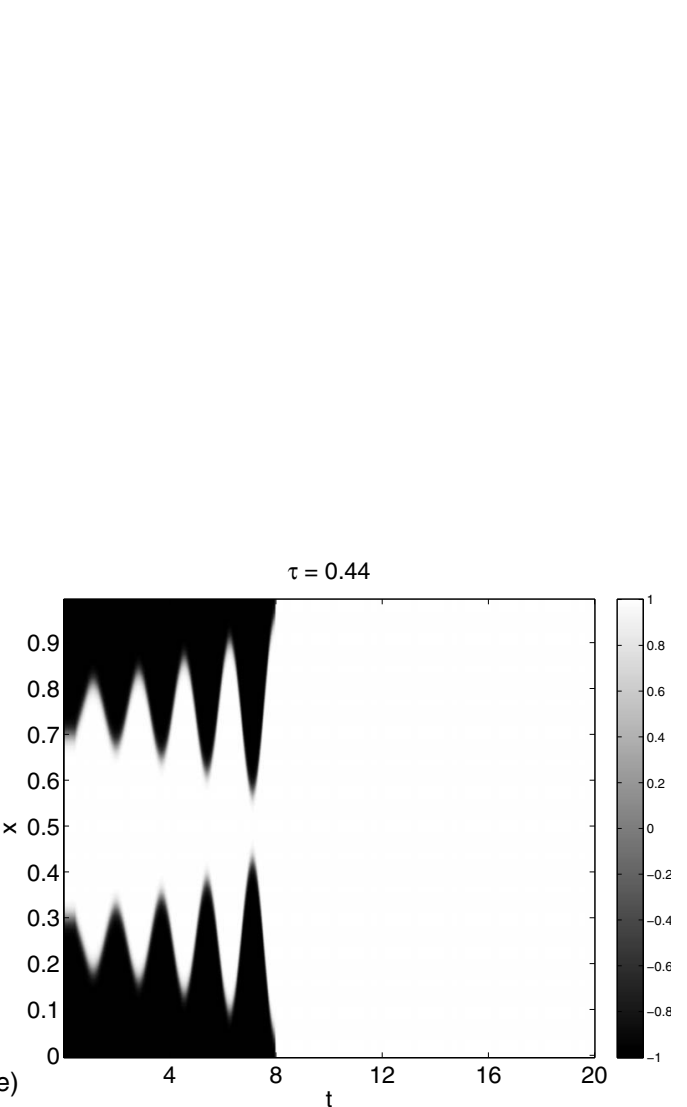

FIG. 6. Evolution of one-dimensional bumps for Eq. (3) for $h=0, \gamma=-1, \epsilon=0.01$ and various values of $\tau$. The gray level codes for values of $\phi$. The interface (or front) corresponds to the small region of fast transition between $\phi=+1$ (white) and $\phi=-1$ (black). The values of $\tau$ appearing at the top of each figure are approximation of the values used in our simulations: (a) $\tau=0.0,(\mathrm{~b}) \tau=0.38,(\mathrm{c}) \tau=0.41$, (d) $\tau=0.42$, (e) and $\tau=0.44$.

\section{DYNAMICS OF TWO-DIMENSIONAL FRONTS WITH RADIAL SYMMETRY}

The evolution of the radius $\rho$ of a two-dimensional front with radial symmetry in a domain $\Omega$ with $|\Omega|=1$ is given by

$$
\rho_{t}=-\frac{1}{\rho}-h-\gamma\left[1-2 \pi \rho^{2}(t-\tau)\right],
$$

which can be obtained from Eq. (19) by disregarding angular variations $\left(\rho_{\theta}=\rho_{\theta \theta}=0\right)$. We consider an initial radius $\rho(0)=\rho_{0}$ and $\rho(t)=\rho_{-}$for $-\tau<t<0$.
Equation (26) is a first order, nonlinear, delay-differential equation. For $\gamma=0$ and $h=0$ (no global feedback and no potential difference between phases) Eq. (26) is the classical flow by mean curvature equation discussed in Sec. I (background) whose solution describes shrinkage of circles to a point in finite time. The same occurs for $h>0$ with a larger shrinkage time. For $h<0$, Eq. (26) has an unstable steady state $\bar{\rho}=-h$ who acts as a threshold: circles with $\rho<\bar{\rho}$ shrink to a point in finite time while circles with $\rho>\bar{\rho}$ increase unboundedly. Following our convention relating the sign of $h$ and the sign of the phase $(\phi)$, only in this last case the break 
of symmetry in the double-well potential opposes the curvature effects and the system will reach a fully nondepolarized state.

Global inhibition $(\gamma<0)$ opposes curvature effects by a different mechanism. For high enough levels, localized circular fronts arise. Their radii are stable steady states $\bar{\rho}$ of Eq. (26); i.e., $\bar{\rho}$ satisfy

$$
2 \pi \gamma \bar{\rho}^{3}-(h+\gamma) \bar{\rho}-1=0 .
$$

Note that $\bar{\rho}$ is independent of $\tau$.

We investigate the stability properties of $\bar{\rho}$ following standard procedures. We first linearize Eq. (26) around $\bar{\rho}$ by defining $v(t)=\rho-\bar{\rho} \ll 1$. Substitution into Eq. (26) gives

$$
\frac{d v(t)}{d t}=\frac{1}{\bar{\rho}^{2}} v(t)+4 \pi \gamma \bar{\rho} v(t-\tau) .
$$

Next we assume a solution of the form $v(t)=v_{0} e^{\lambda t}$ for Eq. (28). Substituting into Eq. (28) gives the characteristic equation

$$
F(\lambda)=\lambda-\frac{1}{\bar{\rho}^{2}}-4 \pi \gamma \bar{\rho} e^{-\lambda \tau} .
$$

Equation (26) is linearly stable if all the solutions of the characteristic Eq. (29) are negative, and linearly unstable otherwise. Note that unlike the $1 \mathrm{D}$ case, the characteristic Eq. (29) includes the steady state $\bar{\rho}$, and no rescaling similar to Eqs. (22) is possible due to the nonlinearities; i.e., different dynamic situations arise for different values of $\gamma$ and $h$. Note also that although the steady radii $\bar{\rho}$ are independent of the delay parameter $\tau$, the characteristic equation depends on $\tau$, and in consequence the stability properties of $\bar{\rho}$ also depend on $\tau$.

We first consider $\tau=0$ (global feedback with no delay). From Eq. (29), $\bar{\rho}$ is stable if $1+4 \pi \gamma \bar{\rho}^{3}<0$ and unstable $1+4 \pi \gamma \bar{\rho}^{3}>0$. Figure 7 shows radii $\bar{\rho}$ of the steady circular fronts and their stability properties as a function of the global feedback parameter $\gamma$ for various representative values of $h$ (potential difference between the two phases) and $\tau=0$. Solid curves correspond to stable radii $\bar{\rho}_{s}$ and dashed curves correspond to unstable radii $\bar{\rho}_{u}$. Both $\bar{\rho}_{s}$ and $\bar{\rho}_{u}$ depend on $\gamma$ and $h$. For given values of $h$ and $\gamma$, circles with initial radius below $\bar{\rho}_{u}$ shrink to a point in finite time while circles with initial radius above $\bar{\rho}_{u}$ converge to localized circles with radius $\bar{\rho}_{s}$. For some values of $h$ there is a range of values of $\gamma$ for which no equilibrium radii exist. For this values of $h$ and $\gamma$, circles shrink to a point in finite time independently of their initial radius.

For $\gamma<0$ (negative global feedback) localized solutions emerge as predicted in $[8,65]$. A stable and unstable equilibrium radii coexist within some range of values of $\gamma$ which depends on the value of $h$. It increases with $h$ for values of $h$ small enough [Figs. 7(a)-7(d)] and it decreases with $h$ for higher values of $h$ [Figs. 7(e) and 7(f)]. For $\gamma>0$ (positive global feedback) steady states $\bar{\rho}$ are always unstable (dashed curves) and no localized solutions are possible. However, these unstable radii $\bar{\rho}$ play the role of a threshold radii dividing between regions of shrinkage and unbounded growth even for positive values $h$. In Fig. 8 we illustrate the evolu- tion of the radius of circular fronts for $\tau=0, h=0$ and various representative values of $\gamma$ [included in the diagram presented in Fig. 7(b)]. For low enough levels of global inhibition $(\gamma$ negative and small enough in absolute value), circular fronts shrink to a point in finite time; The shrinkage time increases as global inhibition increases. For $\gamma$ close enough to the knee of the "horizontal parabola" in Fig. 7(b) the shrinkage time approaches its upper bound (solid curve in Fig. 8). As global inhibition increases further, stable radii, corresponding to localized circular fronts, emerge (dashed and dash-dotted curves in Fig. 8).

As the result of global inhibition, fronts that would otherwise shrink to a point in finite time can stabilize into a localized front (separating the two coexisting phases) by crossing a curve of unstable equilibria in Fig. 7. For example, consider a circle with initial radius $\rho_{0}=0.1$. In the absence of inhibition $\gamma=0$ the representative point is located to the right (and below) of the dashed threshold-curve (unstable equilibria) in Fig. 7. Such a circle shrinks to a point in finite time. By increasing global inhibition, this point moves to the left in the equilibrium diagrams in Fig. 7. For a sufficiently small value of $\gamma$ it crosses the threshold (dashed) curve and enters the basin of attraction of the stable equilibrium eventually converging to a localized front. For small enough values of $h$ [Figs. 7(e) and 7(f)] there is a small range of values of $\gamma$ for which an initial radius point will lay above the curve of threshold equilibria (dashed) but not below the curve of stable equilibira (solid). In these cases, the corresponding circles grow eventually reaching the boundaries of the domain. This range of values of $\gamma$ increases with decreasing values of $h$. In this sense, global inhibition acts as a control mechanism for the selection of the stationary phase of the system. However, if $h$ is fixed and high enough, global inhibition alone is not enough to fully reverse the stationary phase; i.e., circles that shrink to a point in finite time can at most stabilize into a localized front but never reach the boundaries of the domain. Below we show that phase reversal can be achieved as the delay times increase above some values causing the stable circular fronts to loose stability.

We now consider values of $\tau>0$ (delayed-time global feedback). The location the steady radii $\bar{\rho}_{s}$ and $\bar{\rho}_{u}$ are as in Fig. 7. However, as $\tau$ departs from zero, their stability properties change. More specifically, stable steady state radii $\left(\bar{\rho}_{s}\right)$ become unstable for large enough values of $\tau$. We illustrate the dynamics of $\rho$ for various representative parameter values in Figs. 9 and 10. In these figures, the horizontal, green and red dashed lines correspond to the unstable and stable radii $\bar{\rho}_{u}$ and $\bar{\rho}_{s}$ respectively. We call $\bar{\Omega} \in \Omega$ the circular domain of radius 0.5 . (The radius of a circular domain $\Omega$ with $|\Omega|=1$ is $\sim 0.564$.) Figure 9 shows the evolution of $\rho$ for $\gamma=-8, h=0$ and $\rho_{-}=\rho_{0}$ (i.e., $\rho=\rho_{0}$ for $t<0$ ) and various representative values of $\tau$. Radii initially below the unstable radius $\bar{\rho}_{u}$ decrease to zero in finite time (not shown). In Fig. 9(a), the initial radius $\rho_{0}$ is just above $\bar{\rho}_{u}$. For low enough values of $\tau, \rho$ converges to $\bar{\rho}_{s}$ (not shown). As $\tau$ increases, $\rho$ displays damped oscillations eventually converging to $\bar{\rho}_{s}$. Figigure 9, top-left panel, shows the first few periods. (Note that the oscillations' amplitude decreases very slowly with time.) The initial amplitude of these oscillations increases 

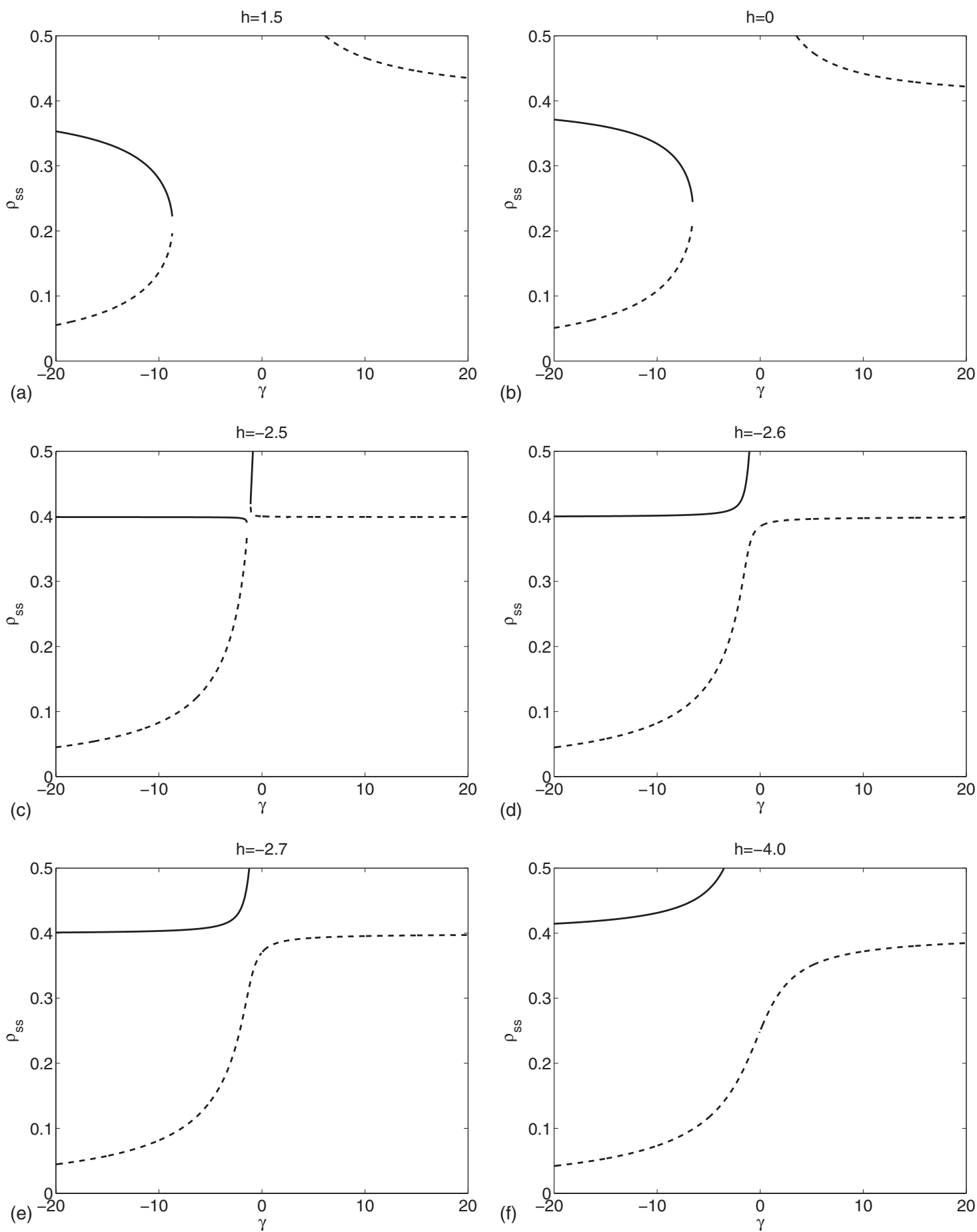

FIG. 7. Radii of the equilibrium circular fronts $\rho_{s s}(\bar{\rho})$ as a function of the global feedback parameter $\gamma$ for various values of $h$ : (a) 01.5 , (b) 0.9 , (c) 2.5, (d) 2.6, (e) 2.7, (f) 4.0, and $\tau=0$ (no delay). Full and dashed curves correspond to stable and unstable circular fronts, respectively.

with $\tau$. For the value of $\tau$ corresponding to the top-right panel, the initial amplitude is maximal within $\bar{\Omega}$ (a slightly higher value of $\tau$ such the one corresponding to the leftbottom panel brings the solution outside $\bar{\Omega}$ ). After $\rho$ turns around, it decreases and, since $\left|0.5-\bar{\rho}_{s}\right|$, is larger than $\left|\bar{\rho}_{s}-\bar{\rho}_{u}\right|(\rho=0.5$ is the boundary of $\bar{\Omega})$, it crosses $\bar{\rho}_{u}$. Once this happens, $\rho$ decreases to zero in finite time as it occurs for initial radii below $\bar{\rho}_{u}$. Note that the upper steady radius $\bar{\rho}_{s}$ does not need to loose stability in order for $\rho$ to cross the lower steady radius $\bar{\rho}_{u}$. Oscillations may still be damped but if their initial amplitude, determined by $\left|0.5-\bar{\rho}_{s}\right|$, is larger than $\left|\bar{\rho}_{s}-\bar{\rho}_{u}\right|$, then $\rho$ crosses the unstable radius $\bar{\rho}_{u}$ before being able to turn around. Note also that $\rho$ may display multiple oscillations before crossing $\bar{\rho}_{u}$. For a slightly larger value of $\tau$ (Fig. 9(a), left-bottom panel) the initial amplitude of the oscillation allows $\rho$ to escape $\bar{\Omega}$ before turning around. 

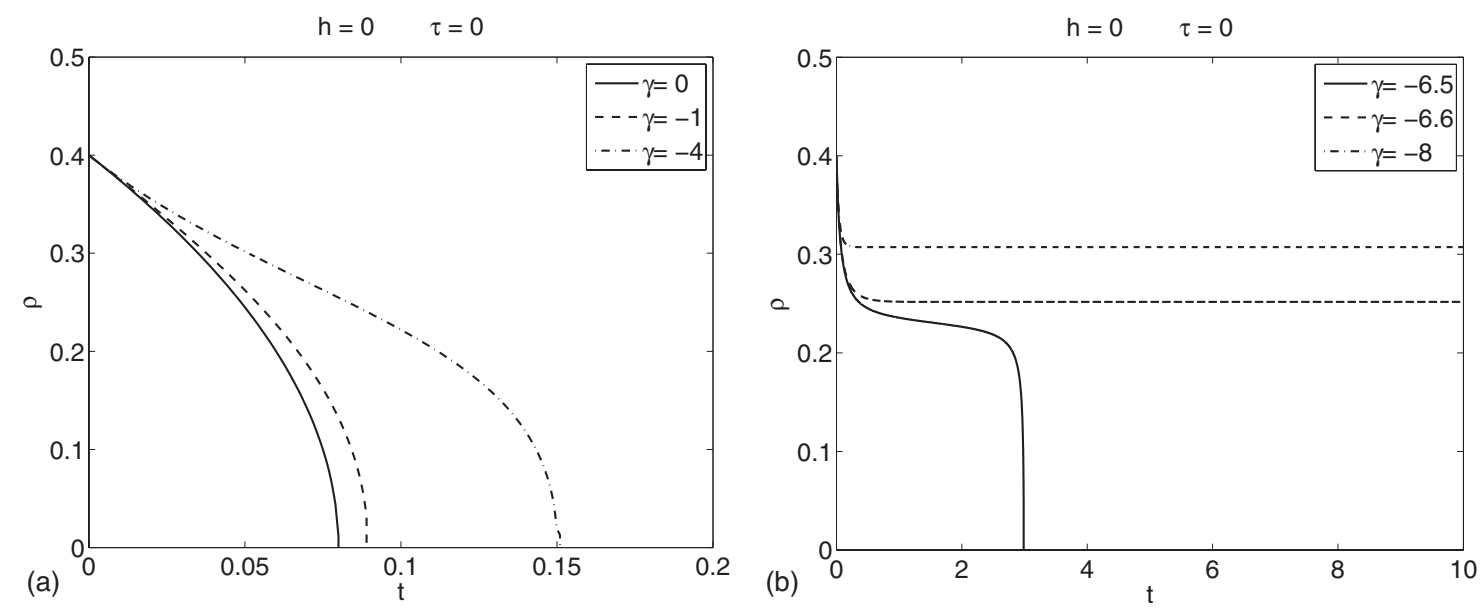

FIG. 8. Evolution of radii $\rho$ of circular fronts for various values of the global feedback parameter $\gamma, h=0$ (two phases with equal potential) and $\tau=0$ (no delay).

For larger values of $\tau, \rho$ escapes $\bar{\Omega}$ faster (not shown). The dynamics described in these panels is robust to changes in parameters provided the initial radius $\rho_{0}$ lies between $\bar{\rho}_{u}$ and $\bar{\rho}_{s}$. We refer to the smallest value of $\tau$ such that $\rho$ escapes $\bar{\Omega}$ as $\tau_{\text {rev }}$. In Fig. 9(a) (bottom-right panel) we present graphs $\tau_{\text {rev }}$ as a function of the initial radius $\rho_{0}$ for various representative values of $\gamma$. In all cases $\tau_{r e v}$ is an increasing function of $\rho_{0}$ since the initial oscillations amplitude decreases as $\rho_{0}$ approaches $\bar{\rho}_{s}$. This panel also shows that inhibitory global feedback facilitates the escape since $\tau_{\text {rev }}$ decreases with increasing values of $|-\gamma|$.

This scenario changes for initial radii $\rho_{0}$ above $\bar{\rho}_{s}$ [Fig. 9(b)]. A slight increase in $\tau$ causes the initial amplitude of the damped oscillation (left panel) to be large enough so that $\rho$ crosses $\bar{\rho}_{u}$ before being able to turn around (right panel), and thus decreases to zero in finite time. A significant difference between the two cases described in Figs. 9(a) and 9(b) $\left(\rho<\bar{\rho}_{u}\right.$ and $\left.\rho_{0}>\bar{\rho}_{u}\right)$ is the fact that $\rho(t)$ is initially increasing in the former case and decreasing in the latter case. This is a consequence of having considered $\rho(t)=\rho_{0}$ for $t<0$. More specifically, initially, $\rho_{t}(t)=\left[2 \pi \gamma \rho_{0}^{3}-(h+\gamma) \rho-1\right] \rho_{0}^{-1}$. From Eq. (27), the numerator vanishes for $\rho_{0}=\bar{\rho}_{s}$. It is positive for $\rho_{0}<\bar{\rho}_{s}$ and negative for $\rho_{0}>\bar{\rho}_{s}$.

One strategy to change the direction of motion of circular fronts with radius above $\bar{\rho}_{s}$ is to change the initial conditions to $\rho(t)=0$ for negative values of $t$. In this case, initially,

$$
\rho_{t}(t)=-\frac{1}{\rho_{0}}-h-\gamma .
$$

If $\rho_{0}>-(h+\gamma)^{-1}$, then $\rho(t)$ is initially increasing. We illustrate this in Fig. 10 (with $\gamma$ and $h$ as in Fig. 9). For $\rho_{0}<\bar{\rho}_{s}$ [Fig. 10(a)], the dynamics is similar to Fig. 9(a), and $\rho$ escapes $\bar{\Omega}$ for large enough values of $\tau$. Differently from the previous case, $\rho$ initially increases for $\rho_{0}>\bar{\rho}_{s}$ instead of decreasing [Fig. 10(b)]: for large enough values of $\tau$ the evolution of $\rho$ will be governed by Eq. (30) with $\rho_{0}$ substituted by $\rho$. Provided $\gamma$ is large enough, $\rho$ will increase eventually escaping $\bar{\Omega}$.
A second strategy to change the direction of motion of circular fronts with radius above $\bar{\rho}_{s}$ is to increase the magnitude of global inhibition (decrease $\gamma$ ). Since $\bar{\rho}_{s}$ is a decreasing function of $\gamma$ (see Fig. 7), the relative position of an initial radius with respect to $\bar{\rho}_{s}$ can be changed (from above to below) by decreasing the value of $\gamma$. Circular fronts with initial radii below $\bar{\rho}_{u}$ are not affected by changes in $\tau$ since they are not in the basin of attraction of $\bar{\rho}_{s}$. However, then can be brought to that basin of attraction by changing either the magnitude of global inhibition or, if possible, the value of $h$ (see Fig. 7).

The dynamics described here can be qualitatively predicted by investigating the behavior of the characteristic polynomial $F(\lambda)$ given by Eq. (29). We illustrate the analysis here for $\gamma=-10$ and $h=0$. For these parameter values $\bar{\rho}_{s}=0.334$ and $\bar{\rho}_{u}=0.108$.

Figure 11(a) shows the graphs of $F(\lambda)$ corresponding to these parameters. For $\bar{\rho}=0.108$ (right panel) $F(\lambda)$ has positive roots for all values of $\tau$; i.e., $\bar{\rho}=0.108$ is always unstable. For $\bar{\rho}=0.334$ (left panel) $F(\lambda)$ has a single negative root for $\tau=0$ explaining the stability of $\bar{\rho}_{s}$. As $\tau$ increases, $F(\lambda)$ develops a minimum $\lambda_{\text {min }}$ with an increasing value of $F\left(\lambda_{\text {min }}\right)$. For $\lambda$ for $\tau<\tau_{c} \sim 0.01 F(\lambda)$ has two negative roots and $\bar{\rho}$ is stable; i.e., the localized solution persists. For $\tau>\tau_{c}, F(\lambda)$ has no real roots $\left[F\left(\lambda_{\text {min }}\right)>0\right]$. In this case oscillatory solutions are possible. These oscillatory solutions may be stable $\tau>\tau_{H}$ or unstable.

In order to show that our results are not an artifact of the asymptotic approximation, in Figs. 12 and 13 we present the results of direct simulations of Eq. (3) (two-dimensional case) for $\gamma=-12, h=0, \epsilon=0.01$ and various values of $\tau$. We used $f(\phi)=\left(\phi-\phi^{3}\right) / 2$ (prototypical case) and we choose various representative values of $\gamma$ and $\tau$. We considered a square domain $\Omega=[0,1] \times[0,1](|\Omega|=1)$ with Neuman boundary conditions. We used a forward finite difference scheme with a discretization of 100 points for both the $x$ and $y$ directions. In order to set the initial conditions we have considered a circle $\mathcal{C}$ centered at the point $(0.5,0.5)$ with radius $r=0.3$, and we have defined 

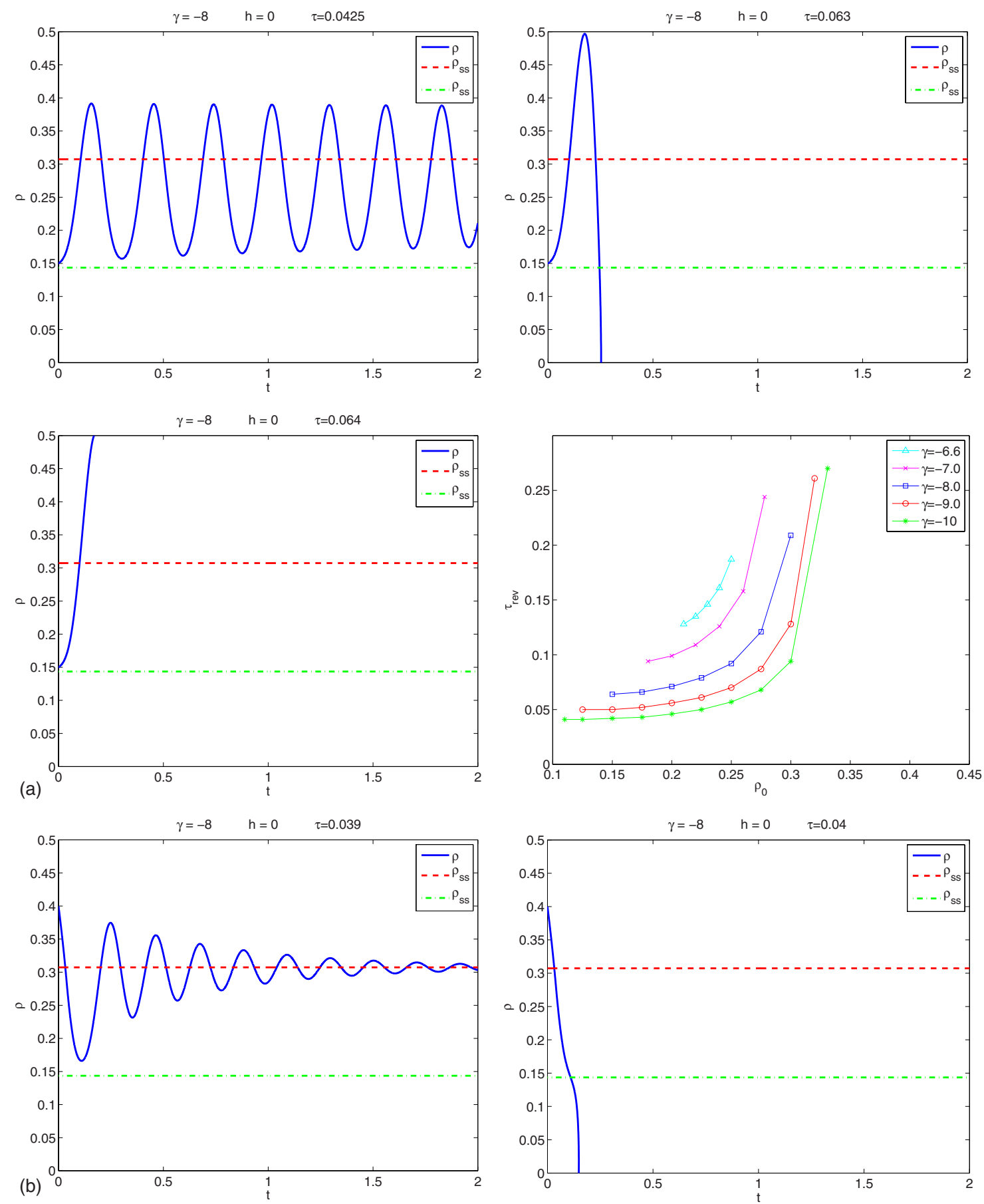

FIG. 9. (Color online) Evolution of the radius $\rho$ of a circular front for various delay times $(\tau), \gamma=-8, h=0$, and $\rho(t)=\rho_{0}$ for $-\tau<t<0$. The stable and unstable steady states corresponding to $\tau=0$ are $\rho_{s s}=0.3073$ and $\rho_{s s}=0.1435$, respectively. (a) Initial radius $\rho_{0}=0.15$. The right-bottom panel shows the phase reversal delay times $\tau_{\text {rev }}$ for various values of $\rho_{0}$ and $\gamma$. (b) Initial radius $\rho_{0}=0.4$.

$$
\phi(x, y, t=0):= \begin{cases}-1 & \text { if }(x, y) \in \mathcal{C} \\ 1 & \text { if }(x, y) \in \Omega \backslash \mathcal{C} .\end{cases}
$$

Note that due to computational limitations, the mesh we used here is less refined (less discretization points per unit of length) than the one used in the $1 \mathrm{D}$ case (100 points).

In Figs. 12 and 13, we plot the value of the order parameter $\phi$ (gray level) on the middle line $y=0.5$ (ordinates) as a function of time (abscissa). Figure 12 corresponds to $\tau=0$

(no delay), $h=0$ (no potential difference between phases) and various values of the global feedback parameter $\gamma$. As predicted analytically (see Fig. 8), for $\gamma=0$ an initial circle (blue) shrinks to a point in finite time. As $\gamma$ increases, the shrinkage time also increases. For $\gamma$ above some threshold localized solutions appear. Figure 13 corresponds to $\gamma=-12$, $h=0$ and various values of $\tau$. The localized solutions loose stability in a Hopf-bifurcation and a homogeneous steady solution in the nondepolarized $\phi=-1$ phase emerges. These 

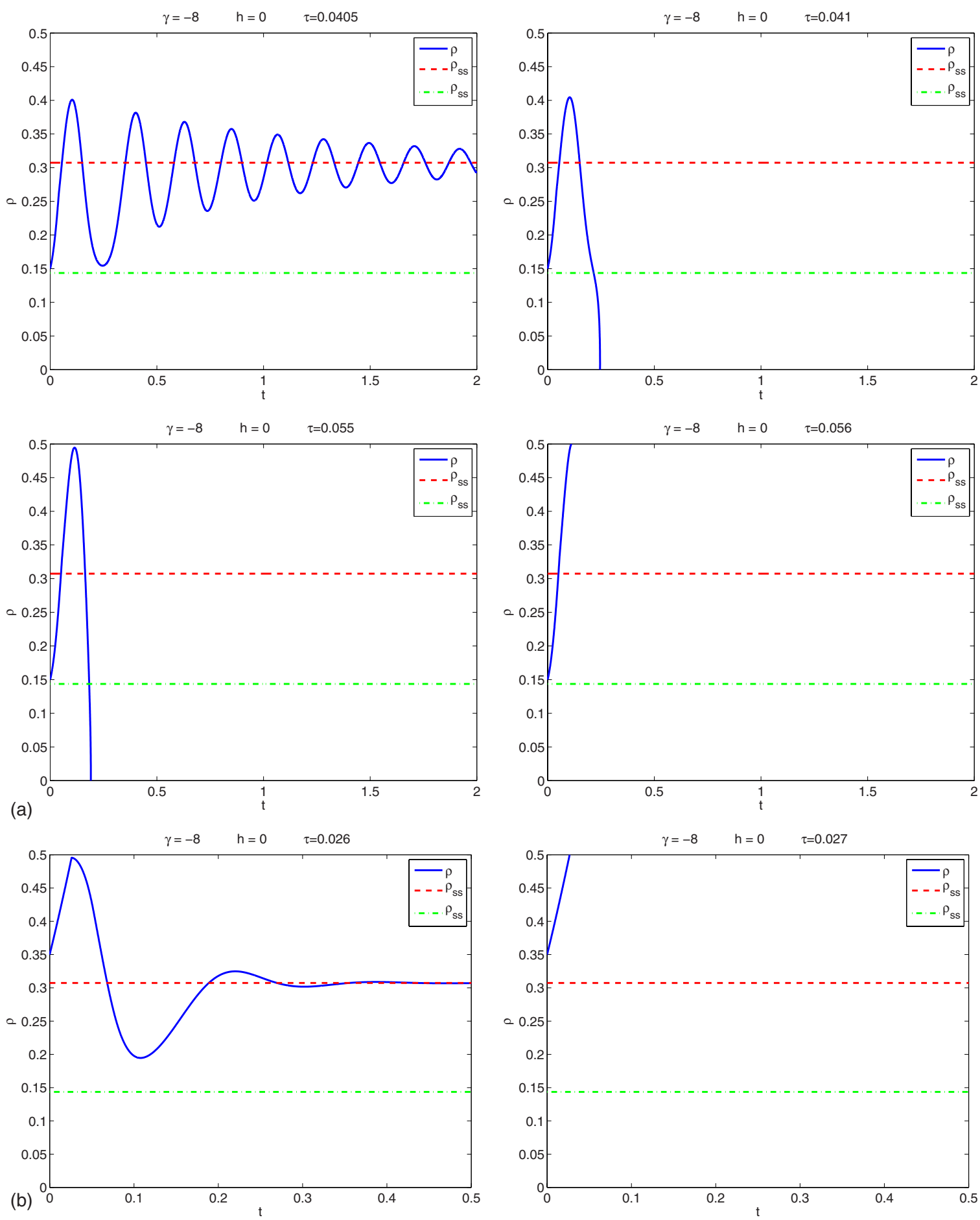

FIG. 10. (Color online) Evolution of the radius $\rho$ of a circular front for various delay times $(\tau), \gamma=-8, h=0$, and $\rho(t)=0.0$ for $-\tau<t<0$. The stable and unstable steady states corresponding to $\tau=0$ are $\rho_{s s}=0.3073$ and $\rho_{s s}=0.1435$, respectively. (a) Initial radius $\rho_{0}=0.15$. The right-bottom panel shows the phase reversal delay times $\tau_{\text {rev }}$ for various values of $\rho_{0}$ and $\gamma$. (b) Initial radius $\rho_{0}=0.35$.

graphs also illustrate the fact that the front structure of the solution is preserved for values of $\gamma<0$ and $\tau>0$ even when the front looses stability. We have obtained similar results using initial two-dimensional fronts with no radial symmetry (not shown). The dynamics and mechanisms explained + here persist when the assumption of radial symmetry is removed.

\section{DISCUSSION}

In this paper, we have investigated the dynamics of oneand two-dimensional fronts in the bistable reaction-diffusion Eq. (3) with delayed-time inhibitory global feedback. This equation, referred to as the Hodgkin-Grafstein (HG) equation, has been used to describe the dynamics of extracellular 

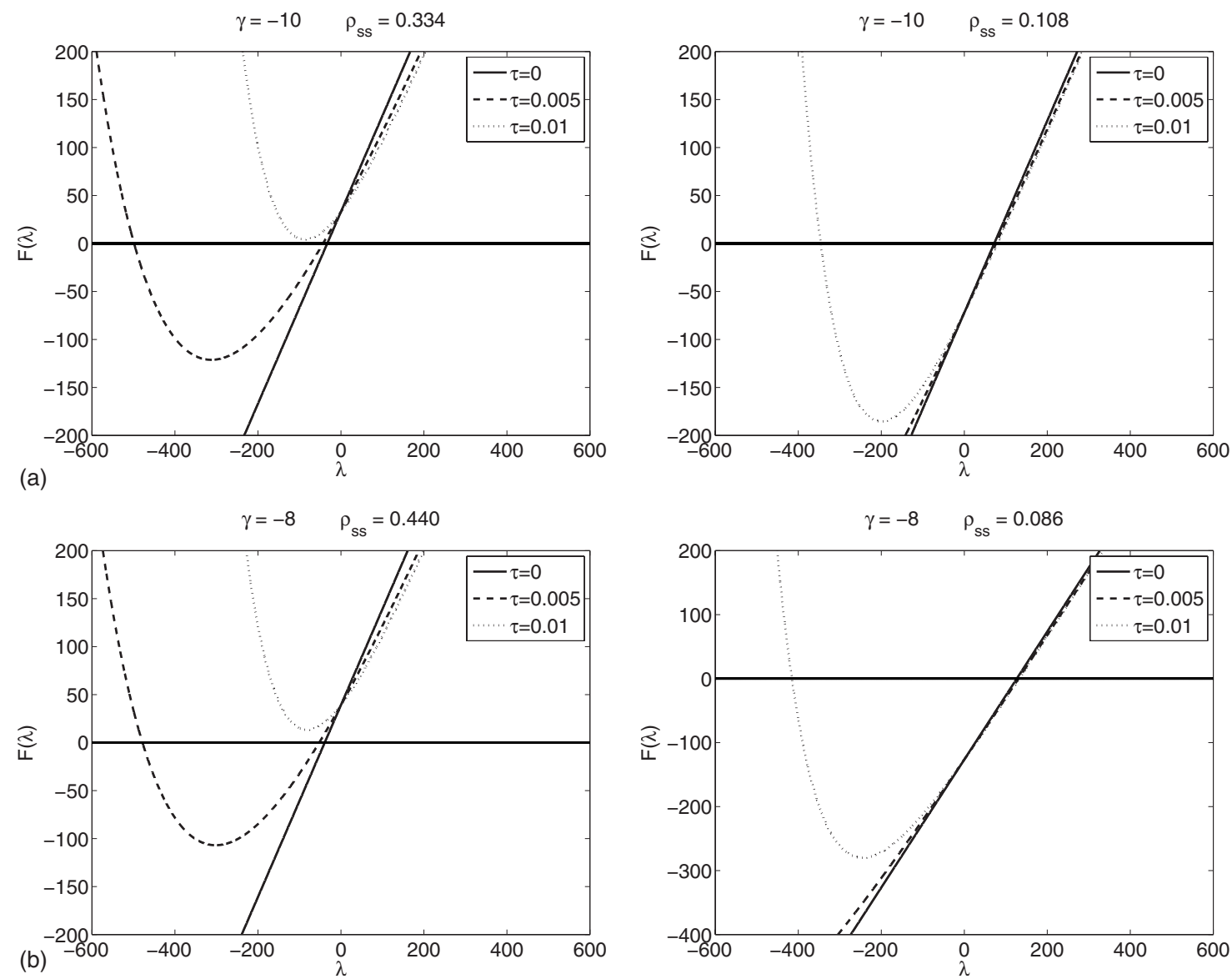

FIG. 11. Characteristic polynomial $F(\lambda)$ as a function of $\lambda$ for two-dimensional circular fronts and two negative values of the global feedback parameter: (a) $\gamma=-10$ and (b) $\gamma=-8$. The left panels correspond to the radii of the stable circular fronts: (a) $\bar{\rho}\left(=\rho_{s s}\right)=0.334$ and (b) $\bar{\rho}\left(=\rho_{s s}\right)=0.440$. The right panels correspond to the radii of the unstable circular fronts: (a) $\bar{\rho}\left(=\rho_{s s}\right)=0.108$ and (b) $\bar{\rho}\left(=\rho_{s s}\right)=0.086$.

potassium ions during spreading depression [37,38]. Although it is an early model, it is an important component in some more detailed models describing this phenomenon $[30,70]$. The sharp transition from a physiological to a pathological state is marked by a front. Understanding the dynamics of these fronts and developing control mechanisms to prevent their spread and, even better, promote shrinkage of the depolarized region (by reversing the front direction) has became an important goals in this field [70] (and references therein). Our work introduces time-delayed global feedback to meet these goals thus complementing previous work by other authors who have used instantaneous (no time-delay) global feedback [8] or local-in-space, time-delayed feedback $[38,56]$ to investigate the HG equation. Our results are also applicable to the study of other phenomena described by the bistable equation such as phase-transition dynamics and the evolution of chemical concentrations $[35,36]$.

It was previously shown that localized fronts can be obtained by applying inhibitory global feedback (nondelayed) $[8,40,65]$. Fronts fail to propage due to global coupling effects. Numerical simulations using Eq. (3) have shown that front structures persist under time-delayed global feedback. Moreover, for appropriate delay times, we found delayedinduced fronts, that is, localized fronts that loose stability and move in a direction opposite to their natural direction corresponding to the absence of global coupling. More spe- cifically, the application of delayed-time global feedback to a front (that otherwise shrinks to a point in finite time) may generate front oscillations that loose stability causing the front to grow unboundedly until it reaches the boundaries of the domain. In order to investigate the nature of these instabilities we used formal asymptotic techniques and derived Eq. (18) governing the evolution of fully developed fronts. Stability analysis shows one-dimensional fronts and twodimensional fronts with radial symmetry loose stability in a Hopf bifurcation. Based on our numerical simulations, we hypothesize the same is true for two-dimensional fronts with other geometries. Front oscillations have been observed in various other fields $[25,35,66,67,71,72]$. In contrast to the results presented here, these oscillations are stable result from the hyperbolicity of the system such as in pulsons and systems with memory $[4,72]$.

The results and control strategies presented in this paper are relevant to other spatially extended pattern forming systems that display bistable dynamics such as the Allen-Cahn and phase-field equations in liquid-solid phase transitions [34,36], and other chemical and biological systems $[35,61,73-78]$ (see also references in the introduction). In some cases, bistability results from the coupling among the equations in the system such as in the FitzHugh-Nagumo model and models of the Belousov-Zhabotinsky reaction (see [23]). In other cases, such as in phase-field models [34], a 

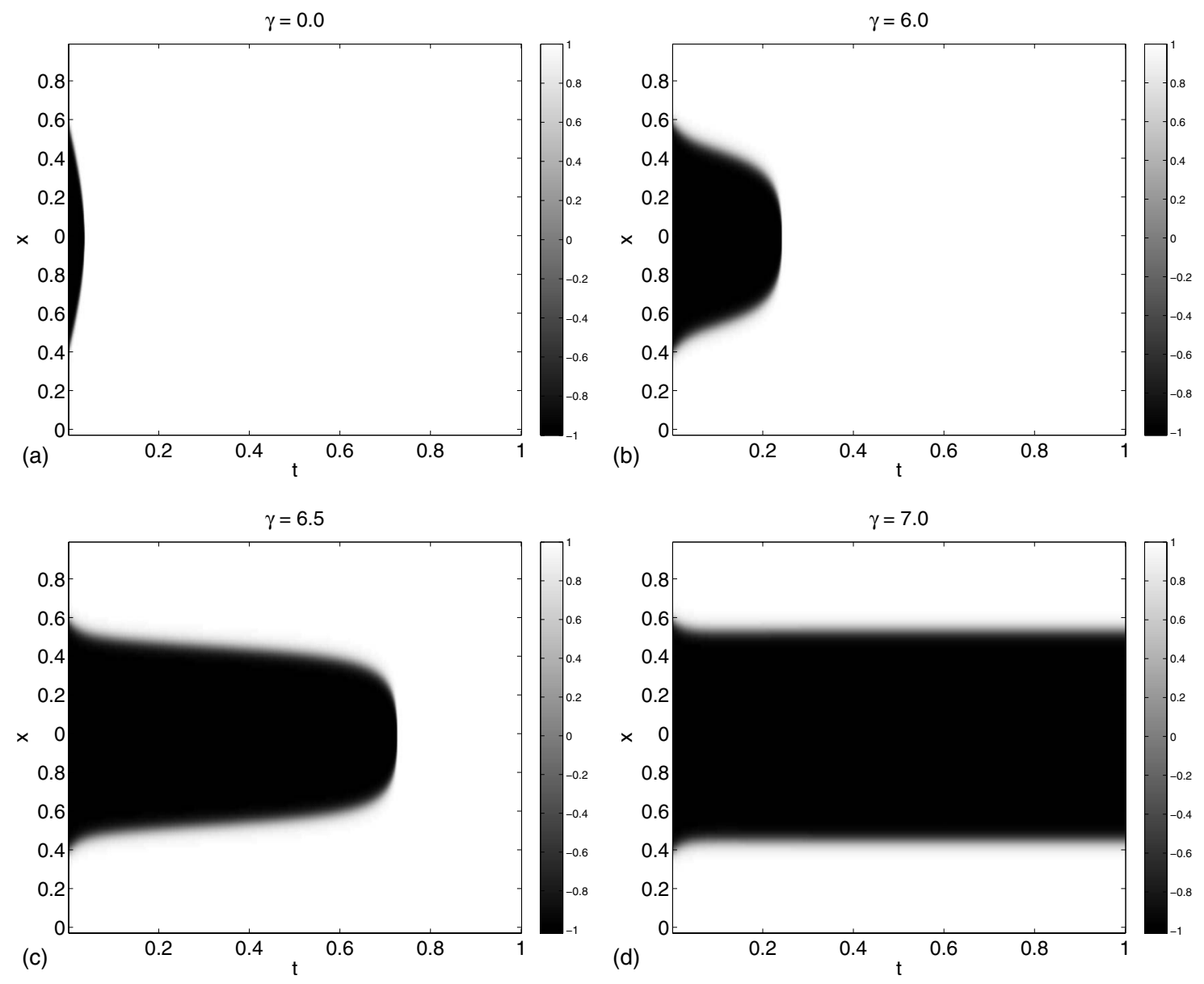

FIG. 12. Evolution of an initially circular front according to Eq. (3) (2D) for $h=0, \tau=0, \epsilon=0.01$ and various values of $\gamma$ : (a) $\gamma=0$, (b) $\gamma=-6.0$, (c) $\gamma=-6.5$, and (d) $\gamma=-7.0$. The gray level codes for values of $\phi$. The interface (or front) corresponds to the small region of fast transition between $\phi=+1$ and $\phi=-1$.

bistable equation describing the evolution of the phase is coupled to a second equation describing the evolution of the temperature but not involved in the generation of bistability. These cases may require a different approach when applying time-delay and instantaneous (no time-delay) global coupling.

Equation (18) generalizes the flow by mean curvature equation which is recovered when $\gamma=0$. The first term corresponds to curvature effects that promote shrinkage of convex curves. In a nonplanar two-dimensional front, the change of direction in front motion depends on the ability of the remaining terms to overcome the curvature effects. In Eq. (18), this is expressed by the existence of stable fixed-points as occurs for Eq. (26). This does not necessarily occur for all types of time-delayed global feedback. Consider, for example, the following time-delay global feedback term

$$
\left\langle\phi_{\tau}\right\rangle-\left\langle\phi_{0}\right\rangle=\frac{1}{|\Omega|} \int_{\Omega}[\phi(x, t-\tau)-\phi(x, t)] d x .
$$

resembling the local feedback term $\phi(t-\tau)-\phi(t)$ used by other authors. The corresponding equation for the evolution of the radius of a two-dimensional front with radial symmetry is given by

$$
\rho_{t}=-\frac{1}{\rho}-h+2 \gamma \pi\left[\rho^{2}(t-\tau)-\rho^{2}(t)\right] .
$$

In this case, the fixed-points for $\gamma=0$ and $\gamma \neq 0$ coincide. Similar results are obtained for one-dimensional fronts.

In this work we have considered linear global feedback. However, similar arguments can be used for other nonlinear global feedback terms $\langle g(\phi)\rangle(t)$ where $g$ is an odd, continuously differentiable function. In this case, $\alpha$ in Eq. (18) should be substituted by $\alpha g(1)$ (not shown). The use of nonlinear global feedback terms may generate a richer dynamics.

In this paper, we have not considered the effects of the interaction between fronts and domain boundaries. It is, in principle, possible that these interactions generate some type of reflection of the interface, specially when the latter is not circular. For the cases we considered, our simulations using the full PDE show that this is not the case. Our results show that persistent oscillatory fronts emerge in this case. Establishing how global feedback, delay, front curvature, and boundary effects combine to generate these localized structures calls for more research. 

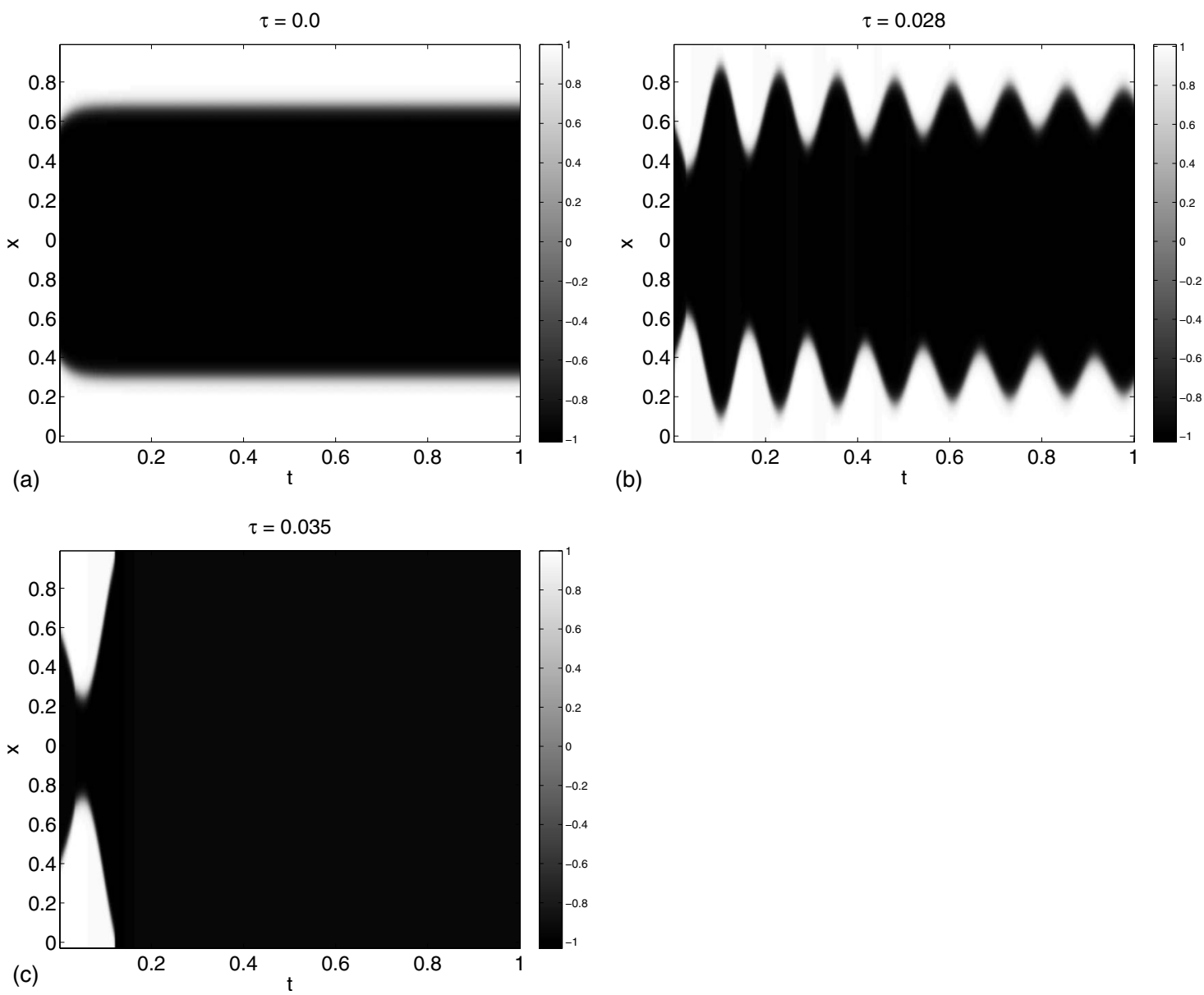

FIG. 13. Evolution of an initially circular front according to Eq. (3) (2D) for $h=0, \gamma=-12, \epsilon=0.01$ and various values of $\tau$ : (a) $\tau=0$, (b) $\tau=0.028$ and (c) $\tau=0.035$. The gray level codes for values of $\phi$. The interface (or front) corresponds to the small region of fast transition between $\phi=+1$ and $\phi=-1$.

\section{ACKNOWLEDGMENTS}

This work was partially supported by NSF Grant No. DMS-0817241 (H.G.R.). Also supported by Grants No. FIS 2009-13370-C02-01 (V.M) and No. 2009 SGR-164 (V.M.).

\section{APPENDIX A: STABILITY ANALYSIS OF ONE- DIMENSIONAL FRONTS}

Here we follow [41] and sketch the stability analysis for the one-dimensional front Eq. (20). We first define $v(t)$ $=s(t)-\bar{s}$ and substitute into Eq. (20) obtaining

$$
\frac{d v(t)}{d t}=2 \gamma v(t-\tau) .
$$

Next we look for solutions of the form $v(t)=v_{0} e^{\lambda t}$. Substituting into Eq. (A1) gives the characteristic equation

$$
F(\lambda)=\lambda-2 \gamma e^{-\lambda \tau}=0 .
$$

Clearly, $\bar{s}$ is stable if of all the solutions to the characteristic Eq. (A2) have negative real part and unstable otherwise. For $\tau=0$ (no delay), $\lambda=2 \gamma$ and $\bar{s}$ is stable (unstable) for $\gamma<0$ $(\gamma>0)$.

For $\tau>0$ and $\gamma>0$ (positive delayed global feedback) Eq. (A2) has a positive root and then $\bar{s}$ is unstable; i.e., no local- ized solutions exist. This can be easily seen by observing that $F(0)=2 \gamma<0$ and $\lim _{\lambda \rightarrow \infty} F(\lambda)=\lambda>0$. Consequently, there exists a value of $\lambda>0$ such that $F(\lambda)=0$.

For $\tau>0$ and $\gamma<0$, standard calculations show that $F(\lambda)$ develops a minimum given by

$$
\lambda_{\min }=-\frac{1}{\tau} \ln \left(-\frac{1}{2 \gamma \tau}\right) .
$$

Substitution into Eq. (A2) gives

$$
F\left(\lambda_{\text {min }}\right)=\frac{1}{\tau}\left[1-\ln \left(-\frac{1}{2 \gamma \tau}\right)\right] .
$$

$F\left(\lambda_{\text {min }}\right)$ vanishes at $\tau_{c}=-(2 \gamma e)^{-1}$ corresponding to $\lambda_{\text {min }}\left(\tau_{c}\right)=2 \gamma e$. If $\tau<\tau_{c}$, then $F\left(\lambda_{\text {min }}\right)<0$. To show this we first calculate

$$
\frac{d}{d \tau} F\left(\lambda_{\text {min }}\right)=\frac{1}{\tau^{2}} \ln \left(-\frac{1}{2 \gamma \tau}\right),
$$

which is positive for $\tau<-(2 \gamma)^{-1}$ and vanishes at $\tau=-(2 \gamma)^{-1}$. Note that $\tau_{c}<-(2 \gamma)^{-1}$. The values of $\lambda_{0}$ for which $F\left(\lambda_{0}\right)=0$ satisfy $\lambda_{0}=2 \gamma e^{-\lambda_{0} \tau}<0$. These values cannot be calculated explicitly. However, the following calculations show that $\lambda_{0} \in(2 \gamma, 2 \gamma e)$, 


$$
\begin{gathered}
F(2 \gamma)=2 \gamma\left(1-e^{-2 \gamma \tau}\right)>0, \\
F(2 \gamma e)=2 \gamma e\left(1-e^{-2 \gamma \tau e-1}\right)>0 .
\end{gathered}
$$

For $\tau>\tau_{c}, F\left(\lambda_{m}\right)>0$ and the characteristic polynomial $F(\lambda)$ has no real roots. To find the complex roots we define $\lambda=\mu+i \omega$. Substituting into the characteristic Eq. (A2) and separating the real and imaginary parts we get

$$
\left\{\begin{array}{c}
\mu=2 \gamma e^{-\mu \tau} \cos (\omega \tau), \\
\omega=-2 \gamma e^{-\mu \tau} \sin (\omega \tau) .
\end{array}\right.
$$

From the first equation in Eq. (A6), if $\omega \tau<\pi / 2$, then $\cos (\omega \tau)>0$ and $u<0$. If $\cos (\omega \tau)>0$ and $\omega \tau<\pi / 2$, then $\omega \tau<\pi / 4$. The second equation in Eq. (A6) is satisfied since $-2 \gamma \tau e^{-u \tau} \sin (\omega \tau)<0$ for $\omega \tau<\pi / 2$.

From the first equation in Eq. (A6), $u=0$ for $\omega \tau=\pi / 2$, and from the second equation in Eq. (A6), this corresponds to $\tau=\tau_{H}$ given by

$$
\tau_{H}=-\pi /(4 \gamma)
$$

Note that $\tau_{c}<\tau_{H}$. Note also that for values of $\tau \in\left(\tau_{c}, \tau_{H}\right)$, $\mu<0$. Assume that $\mu>0$, then, from the first equation in Eq. (A6), $\omega \tau>\pi / 2$ (restricted to the first two quadrants). So, from the second equation in Eq. (A6) and since $e^{-u \tau}<1$ and $\sin (\omega \tau)<1, \omega \tau<-2 \gamma \tau<-2 \gamma \tau_{H}=\pi / 2$, which is a contradiction.
[1] V. K. Vanag, L. Yang, M. Dolnik, A. M. Zhabotinsky, and I. R. Epstein, Nature (London) 406, 389 (2000).

[2] V. K. Vanag, A. M. Zhabotinsky, and I. R. Epstein, J. Phys. Chem. A 104, 11566 (2000).

[3] L. Yang, M. Dolnik, A. M. Zhabotinsky, and I. R. Epstein, Phys. Rev. E 62, 6414 (2000).

[4] H. G. Rotstein, N. Kopell, A. M. Zhabotinsky, and I. R. Epstein, SIAM J. Appl. Math. 63, 1998 (2003).

[5] H. G. Rotstein, N. Kopell, A. M. Zhabotinsky, and I. R. Epstein, J. Chem. Phys. 119, 8824 (2003).

[6] S. Kawaguchi and M. Mimura, SIAM J. Appl. Math. 59, 920 (1998).

[7] F. Mertens, R. Imbihl, and A. Mikhailov, J. Chem. Phys. 99, 8668 (1993).

[8] L. M. Pismen, J. Chem. Phys. 101, 3135 (1994).

[9] M. Sheintuch and O. Nekhamkina, in Pattern Formation in Continuous and Couple Systems (IMA Volumes in Mathematics and its Applications), edited by M. Golubitsky, D. Luss, and S. H. Strogatz (Springer-Verlag, Berlin, 1999), Vol. 115, p. 265.

[10] U. Middya, D. Luss, and M. Sheintuch, J. Chem. Phys. 100, 3568 (1994).

[11] I. Savin, O. Nekhamkina, and M. Sheintuch, J. Chem. Phys. 115, 7678 (2001).

[12] U. Middya and D. Luss, J. Chem. Phys. 100, 6386 (1994).

[13] F. Plenge, P. Rodin, E. Schöll, and K. Krischer, Phys. Rev. E 64, 056229 (2001).

[14] W. Wang, I. Z. Kiss, and J. L. Hudson, Chaos 10, 248 (2000).

[15] M. Bertram and A. S. Mikhailov, Phys. Rev. E 63, 066102 (2001).

[16] M. Bertram, C. Beta, M. Pollmann, A. S. Mikhailov, H. H. Rotermund, and G. Ertl, Phys. Rev. E 67, 036208 (2003).

[17] M. Bertram and A. S. Mikhailov, Phys. Rev. E 67, 036207 (2003).

[18] M. Pollmann, M. Bertram, and H. H. Rotermund, Chem. Phys. Lett. 346, 123 (2001).

[19] H. Riecke, in Pattern Formation in Continuous and Coupled Systems (IMA Volumes in Mathematics and its Applications), edited by M. Golubitsky, D. Luss, and S. H. Strogatz (Springer-Verlag, Berlin, 1999), Vol. 115, p. 215.

[20] S. H. Strogatz and R. E. Mirollo, Phys. Rev. E 47, 220 (1993).

[21] K. Y. Tsang, R. E. Mirollo, S. H. Strogatz, and K. Wiesenfeld,
Physica D 48, 102 (1991).

[22] D. Golomb, D. Hansel, B. Shraiman, and H. Sompolinsky, Phys. Rev. A 45, 3516 (1992).

[23] S. H. Strogatz, Nonlinear Dynamics and Chaos (AddisonWesley Publishing Company, Reading, MA, 1997).

[24] A. A. Nepomnyashchy, A. A. Golovin, V. Gubareva, and V. Panfilov, Physica D 199, 61 (2004).

[25] M. Meixner, P. Rodin, and E. Scholl, Phys. Rev. E 58, 2796 (1998).

[26] D. Golomb and J. Rinzel, Physica D 72, 259 (1994).

[27] D. Golomb and J. Rinzel, Phys. Rev. E 48, 4810 (1993).

[28] D. Terman and D. L. Wang, Physica D 81, 148 (1995).

[29] D. L. Wang and D. Terman, IEEE Trans. Neural Netw. 6, 283 (1995).

[30] F. M. Schneider, E. Scholl, and M. A. Dahlem, Chaos 19, 015110 (2009).

[31] H. G. Rotstein and R. Kuske, Physica D 215, 46 (2006).

[32] M. Tlidi, M. Taki, and T. Kolokolnikov, Chaos 17, 037101 (2007).

[33] N. Akhmediev and A. Ankiewicz, Lecture Notes in Physics Vol. 751 (Springer, Berlin, 2008).

[34] P. C. Fife, Dynamics of Internal Layers and Diffusive Interfaces, Regional Conference Series in Applied Mathematics (Springer-Verlag, Berlin, 1988).

[35] J. D. Murray, Mathematical Biology (Springer, Berlin, 1989).

[36] S. M. Allen and J. W. Cahn, Acta Metall. Mater. 27, 1085 (1979).

[37] B. Grafstein, in Brain Function: Cortical Excitability and Steady Potentials, edited by M. A. B. Brazier (University of California Press, Berkeley, 87, 1963).

[38] M. A. Dahlem, R. Graf, A. J. Strong, J. P. Dreier, Y. A. Dahlem, M. Sieber, H. W. K. Podoll, and E. Scholl, Physica D 239, 889 (2010).

[39] H. G. Rotstein, I. Mitkov, A. M. Zhabotinsky, and I. R. Epstein, Phys. Rev. E 63, 066613 (2001).

[40] H. G. Rotstein, A. M. Zhabotinsky and I. R. Epstein, Phys. Rev. E 74, 016612 (2006).

[41] T. Erneux, Applied Delay Differential Equations (Springer, New York, 2009).

[42] B. Y. Rubinstein, A. A. Nepomnyashchy, and A. A. Golovin, Phys. Rev. E 75, 046213 (2007). 
[43] L. G. Stanton and A. A. Golovin, Phys. Rev. E 76, 036210 (2007).

[44] A. G. Balanov, N. B. Janson, and E. Scholl, Physica D 199, 1 (2004).

[45] A. G. Balanov, V. Beato, N. B. Janson, H. Engel, and E. Scholl, Phys. Rev. E 74, 016214 (2006).

[46] M. Kim, M. Bertram, M. Pollmann, A. von Oertzen, A. S. Mikhailov, H. H. Rotermund, and G. Ertl, Science 292, 1357 (2001).

[47] T. V. Savina, A. A. Nepomnyashchy, and A. A. Golovin, J. Cryst. Growth 307, 490 (2007).

[48] K. Pyragas, Phys. Lett. A 170, 421 (1992).

[49] O. Beck, A. Amann, E. Scholl, J. E. S. Socolar, and W. Just, Phys. Rev. E 66, 016213 (2002).

[50] D. Huber and L. S. Tsimring, Phys. Rev. Lett. 91, 260601 (2003).

[51] D. Huber and L. S. Tsimring, Slow Dynamics in Complex Systems: 3rd International Symposium, edited by M. Tokuyama and I. Oppenheim (2004), p. 448.

[52] G. Kozyreff, A. G. Vladimirov, and P. Mandel, Phys. Rev. Lett. 85, 3809 (2000).

[53] M. Tlidi, A. G. Vladimirov, D. Peroux, and D. Turaev Phys. Rev. Lett. 103, 103904 (2009).

[54] S. Coombes and C. R. Laing, Physica D 238, 264 (2009).

[55] V. K. Jirsa, Philos. Trans. R. Soc. London, Ser. A 367, 1131 (2009).

[56] T. Erneux, G. Kozyreff, and M. Tlidi, Philos. Trans. R. Soc. London, Ser. A 368, 483 (2010).

[57] V. Jasaitis, F. Ivanauskas, and R. Bakanas, Nonlinear Analysis: Modelling and Control 13, 433 (2008).

[58] V. Jirsa (unpublished).
[59] M. Camperi and X.-J. Wang, J. Comput. Neurosci. 5, 383 (1998).

[60] X.-J. Wang, J. Neurosci. 19, 9587 (1999).

[61] P. Coullet, Int. J. Bifurcation Chaos Appl. Sci. Eng. 12, 2445 (2002).

[62] J. Rubinstein, P. Sternberg, and J. B. Keller, SIAM J. Appl. Math 49, 116 (1989).

[63] M. Gage and R. S. Hamilton, J. Diff. Geom. 23, 69 (1986).

[64] M. A. Grayson, J. Diff. Geom. 26, 285 (1987).

[65] H. Sakaguchi, Phys. Rev. E 64, 047101 (2001).

[66] H. G. Rotstein and A. A. Nepomnyashchy, Physica D 136, 245 (2000).

[67] H. G. Rotstein, A. Domoshnitsky, and A. A. Nepomnyashchy, Physica D 146, 137 (2000).

[68] J. W. Thomas, Numerical Partial Differential Equations: Finite Difference Methods (Springer-Verlag, New York, 1995).

[69] S. Coombes, Biol. Cybern. 93, 91 (2005).

[70] R. M. Miura, H. Huang, and J. J. Wylie, Eur. Phys. J. Spec. Top. 147, 287 (2007).

[71] M. Meixner, P. Rodin, and E. Scholl, Phys. Rev. E 58, 5586 (1998).

[72] P. L. Christiansen, N. Gronbech-Jensen, P. S. Lomdahl, and B. A. Malomed, Phys. Scr. 55, 131 (1997).

[73] J. J. Tyson and J. P. Keener, Physica D 32, 327 (1988).

[74] F. Sagués and I. R. Epstein, Dalton Trans. 2003, 1201.

[75] W. van Saarloos, Phys. Rep. 386, 29 (2003).

[76] G. T. Dee and W. van Saarloos, Phys. Rev. Lett. 60, 2641 (1988).

[77] I. R. Epstein and K. Showalter, J. Phys. Chem. 100, 13132 (1996).

[78] V. Méndez and A. Compte, Physica A 260, 90 (1998). 